\title{
Abstracts del XXXIV Congresso Nazionale EDTNA/ERCA Riccione, 4-6 Aprile 2016
}

\section{LE DIVERSE FRONTIERE DEL TRATTAMENTO DIALITICO}

\section{8}

LA LEAN LAB. LA SANITÀ SNELLA NELLA GESTIONE DELLE MALATTIE CRONICHE

Antonelli $D$.

Ospedale Generale Regionale F. Miulli, Andria (BA)

Obiettivo: Utilizzare uno strumento che permetta un'individuazione veloce della diagnosi infermieristica e dei relativi interventi con criteri di appropriatezza definiti e condivisi.

L'Italia è ai primissimi posti al mondo per longevità della popolazione. Poter invecchiare è, senza dubbio, un privilegio della specie umana, ma diventa, al contempo, una sfida per la nostra società, che deve tendere alla migliore qualità possibile degli anni di vita aggiunti. Si definisce oggi "arbitrariamente" anziana e, quindi, potenzialmente paziente geriatrico la persona che ha superato l'età di 65 anni. È evidente che la crescita esponenziale di pazienti di tale fascia d'età e oltre pone problemi di sostenibilità di tutto il sistema sanitario. Partendo da argomenti riguardanti prevenzione, stili di vita, esercizio fisico e importanza della dieta, che rappresentano soluzioni fondamentali per tamponare in tempo situazioni che si sviluppano già in età giovanile/adulta per poi manifestarsi in tutta la loro gravità nell'età geriatrica, si arriva, quest'anno, ad affrontare patologie di grosso impatto, come l'insufficienza renale cronica.

II modello Toyota sulla gestione degli sprechi e sul guadagno dei tempi ha portato, negli ultimi anni, a sviluppare un cambiamento culturale diverso con ottimi risultati in termini di lavoro del personale sanitario dedicato alle malattie croniche. La qualità delle cure è la principale responsabilità del management.

Migliorare l'efficienza e l'efficacia delle prestazioni, riducendo i costi e ottimizzando l'allocazione delle risorse, migliorare la produttività, identificando gli elementi di spreco, e analizzare con metodo scientifico i processi. Il processo di lavoro e l'applicazione della Lean in Sanità hanno portato al miglioramento della qualità dei servizi e del team lavorativo.

043

IOT (INTERNET OF THINGS) E LE IMPLICAZIONI NELL'ASSISTENZA E SISTEMI DI BIOFEEDBACK

Lerose $G$.

P.O. ASM Basilicata, Matera

Introduzione: Internet of Things o, in breve, loT è quell'insieme di tecnologie che portano intelligenza agli oggetti, facendo sì che questi comunichino con noi o con altre macchine, offrendoci un nuovo livello di interazione o di informazione rispetto all'ambiente in cui questi oggetti si trovano. L'Internet delle Cose nel settore sanitario cambierà alla radice il rapporto fra personale sanitario e paziente. Applicazioni e benefici a $360^{\circ}$ dagli anziani e dai malati cronici ai bambini, oltre a una grande diffusione di oggetti wearable che guarda anche alla prevenzione e a un target più giovanile e salutista.

Esempi? Scarpe da ginnastica che trasmettono la velocità di corsa dell'atleta e il suo stato di affaticamento, flaconi delle medicine che ci segnalano quando ci si dimentica di prendere un farmaco, pompe di insulina autoregolanti, piante che comunicano all'annaffiatoio quando è il momento di essere innaffiate, dispenser per pillole automatici precaricati dai medici di base. Una lunga lista di op- portunità e potenzialità che migliorano e possono aiutare anche gli infermieri. Opportunità: Un settore come quello dell'loT deve sicuramente suscitare interesse soprattutto verso gli infermieri che operano con Device estremamente all'avanguardia cercando di sfruttare al massimo le opportunità che le nuove smart technologies portano in merito a raccolta dati, terapie, monitoraggio e così via.

Ormai lo sappiamo, l'Internet of Things si basa sulla creazione di oggetti smart, capaci, cioè, di rivelare dati e comunicare queste informazioni in una rete condivisa. Questa è l'idea di base che nasconde già di per sé parecchie potenzialità. L'evoluzione, quello a cui più si sta lavorando ora, è la possibilità di aumentare sempre più le interazioni tra questi oggetti. L'obiettivo è la creazione di oggetti smart che, tramite sensori, captino dati, li comunichino a una rete e li condividano con altri oggetti attraverso uno scambio reciproco. Un'interazione di questo tipo potrebbe veramente rivoluzionare diversi settori, a partire da quello della sanità. Stiamo parlando della possibilità di installare sensori nel letto per monitorare pressione arteriosa e battiti cardiaci o di braccialetti o altri wearable device, in grado di rilevare i parametri vitali e di inoltrare chiamate di emergenza in caso di anomalie, oppure di smart tag, chip inseriti nei vestiti o stampati sulla pelle sempre con funzione di monitoraggio e di terapia.

Discussione: In questo panorama, il Nursing può avere grandi vantaggi, immaginando un'assistenza legata a oggetti che riescono a supportare l'operatore sia nel monitoraggio che nella gestione delle terapie e non solo immaginando un panorama di un futuro non molto lontano fatto di medicazioni che comunicano quando è il momento di sostituirle e di cerotti stampati con biosensori che rilevano parametri bioumorali comunicandoli a un'app che permetta al professionista di controllare e decidere al meglio l'intervento opportuno.

Ci stiamo avvicinando a un concetto di e-health, una sorta di sanità smart e in rete in cui la medicina diventa telemedicina, cioè medicina a distanza. $E$, in questo, la professione infermieristica può avere un ruolo non marginale, mettendo in atto tutta le conoscenze partendo dal capire le potenzialità della tecnologia loT. Rischi: Immaginando già tutto realtà, dobbiamo anche parlare dei rischi, legati soprattutto alla sicurezza dei dati e alla privacy.

In una ricerca dell'Intel Security e Atlantic Council i principali pericoli sono:

- il furto delle informazioni personali

- la manomissione dolosa e intenzionale dei dispositivi medicali

- il deterioramento o i guasti accidentali

Per questo, sempre secondo lo studio, è importante che si prendano al più presto dei provvedimenti che coinvolgano il campo della ricerca, i governi e le aziende produttrici. In particolare si dovrebbe:

- integrare sin da subito reti e sicurezza di dispositivi, per evitare interventi successivi, costosi e difficili da applicare

- approvare e attenersi a standard di sicurezza (o buone pratiche) condivisi a livello mondiale

- incentivare l'innovazione e la ricerca in materia di Internet of Things

- dare più voce ai malati e alle famiglie

Conclusioni: "La sanità in rete rende l'Internet delle Cose molto personale», ha dichiarato Pat Calhoun, Senior Vice Presidente e General Manager, Network Security di Intel Security. "Quando un dispositivo di rete è collegato a una persona, la possibilità di comunicare le informazioni sanitarie consente di migliorare notevolmente l'assistenza sanitaria, ma le conseguenze di compromissioni alla privacy o alla rete sono altrettanto rilevanti. L'intero ecosistema sanitario deve garantire che la sicurezza sia integrata, dal dispositivo alla rete e al data center, per offrire una migliore qualità della vita». In questo 
panorama, l'infermiere ha l'opportunità di offrire il supporto nello sviluppo di queste tecnologie integrandosi con un sistema di esperti che possono costruire su misura e per ogni realtà sia applicazioni che presidi.

Questo è anche il grande passo avanti da compiere per valorizzare ancora di più il supporto di questa professione, proiettandola nel futuro ripensando a una medicina a misura d'uomo con il supporto tecnologico, che è "Internet Of Things".

\section{5}

IL PAZIENTE DIALIZZATO RICOVERATO IN AMBITO NON NEFROLOGICO: DIFFICOLTÀ E COLLABORAZIONI POSSIBILI

Sgreccia M., Domeniconi S., Dardari L., Bini S.

UO Nefrologia e Dialisi AUSL Romagna, A.O.O. di Rimini, Rimini

Introduzione: All'interno dell'Ospedale di Rimini (AUSL della Romagna), i pazienti dializzati vengono ricoverati in Unità Operative diverse per ragioni non strettamente nefrologiche (fratture, interventi programmati, eventi acuti vari, ecc.). Gli infermieri di questi reparti si trovano a dover gestire pazienti complessi, fragili e a elevata comorbidità. Ciò comporta che il personale infermieristico chieda spesso chiarimenti al Centro Dialisi o alla Degenza di Nefrologia, riguardo alla gestione degli aspetti peculiari che il paziente dializzato presenta: accessi vascolari per dialisi (FAV, CVC), dieta, diuresi, terapia e così via. Non è mai stata compiuta in precedenza, nell'Area Organizzativa Omogenea di Rimini, un'indagine relativa alle conoscenze o alle carenze conoscitive degli infermieri sull'argomento, né esiste una guida scritta che indirizzi i comportamenti in questo senso.

Metodologia:

Obiettivi dello studio

a) Indagare le conoscenze o le carenze conoscitive degli infermieri riguardo alle peculiarità del paziente in dialisi

b) Elaborare un opuscolo indirizzato agli infermieri contenente informazioni pratiche riguardo a questo tema

Popolazione:

È rappresentata dagli infermieri delle seguenti U.O. dell'Ospedale di Rimini:

- Medicina d'Urgenza

- Geriatria

- Ortopedia

- ORL

- Chirurgia Generale

- Malattie Infettive

\section{Strumenti:}

- Gruppo di studio e miglioramento formato da uno studente infermiere, un medico nefrologo, un infermiere e il coordinatore infermieristico

- Questionario anonimo indirizzato agli infermieri che indaga sulle aree ritenute maggiormente critiche

Risultati: Degli 83 infermieri che hanno risposto alla nostra indagine, il 50\% dichiara di aver avuto bisogno di interpellare il Centro Dialisi per chiarimenti. In media, nelle diverse unità operative, circa il $40 \%$ degli intervistati non conosce la "manutenzione ordinaria" della FAV e lascerebbe le medicazioni fino a 48 ore dopo la seduta.

Riguardo alla terapia farmacologica, il $40 \%$ degli intervistati ritiene di poterla somministrare al paziente dializzato utilizzando i normali protocolli standard presenti nel proprio reparto, senza curarsi del fatto che antibiotici, antipertensivi o eparine bpm possono interferire con il trattamento o da esso essere inficiati. Rispetto alla dieta, oltre il $60 \%$ degli intervistati è convinto di dover mantenere la stessa dieta ipoproteica che è consigliata in terapia conservativa.

Infine, soprattutto nei reparti chirurgici, l'infermiere è orientato alla valutazione della diuresi come parametro indiretto di eventuali emorragie post-intervento; oltre la metà degli intervistati ritiene, quindi, utile la valutazione della diuresi delle 24 ore in un paziente che, nella maggior parte dei casi, è anurico.

Conclusioni: Il paziente uremico in trattamento dialitico cronico rappresenta oggi il paradigma della fragilità; l'assistenza infermieristica richiesta all'interno delle aree di degenza generaliste può apparire complessa per quegli infermieri che non frequentano l'ambito nefrologico con assiduità: nel futuro prossimo sarà utile, quindi, ricorrere a una guida scritta e a una serie di incontri formativi dedicati proprio a migliorare le conoscenze in questi settori.
019

LO STAFF INFERMIERISTICO NEL MIGLIORAMENTO DEGLI OUTCOME ATTRAVERSO TRAINING, TECNOLOGIA E INDICATORI DI QUALITÀ Olivieri P. ${ }^{1}$, Santoro G. ${ }^{1}$, Favicchio N.L. ${ }^{1}$, Irace $R .{ }^{1}$, Pizzo A. ${ }^{2}$, Moretti M. ${ }^{2}$, Pelliccia F. ${ }^{3}$, Parisotto M.T. ${ }^{3}$

${ }^{1}$ NephroCare Italia, Centro Dialisi Arenaccia, Napoli, Italia; ${ }^{2}$ NephroCare Italia, Napoli, Italia; ${ }^{3}$ Fresenius Medical Care, NephroCare Coordination, Bad Homburg, Germania

Introduzione: Numerosi studi hanno dimostrato l'esistenza di una correlazione, nel paziente in terapia sostitutiva, tra dose dialitica somministrata e mortalità e morbidità dei pazienti. Per evitare gli effetti negativi, talora irreversibili, della dialisi inadeguata è indispensabile il routinario monitoraggio della dose dialitica somministrata.

L'obiettivo di questo studio è stato di migliorare l'adeguatezza dialitica e di incrementare il tempo di dialisi per il $90 \%$ dei nuovi pazienti dopo la fusione di 2 centri dialisi.

Metodologia: A tempo 0 , due centri A (50 pazienti) e B (20 pazienti) si sono uniti. Agli infermieri è stato richiesto un maggiore controllo della durata della seduta e dell'adeguatezza dialitica dei pazienti B. Dopo 6 mesi dall'accorpamento, lo staff inizia il progetto per il miglioramento dei target clinici attraverso la formazione dei pazienti e pianificando riunioni periodiche per il controllo dei target attraverso le valutazioni di tutti gli indicatori di qualità. Risultati: Per l'analisi statistica è stato utilizzato il T-Test.

II 90\% dei pazienti "B" ha raggiunto il target della durata del tempo di trattamento, con un miglioramento statisticamente significativo $(\mathrm{P}<0.0001) \mathrm{da}$ $225.00 \mathrm{~min}$ (TO) a $239.45 \mathrm{~min}$ (T1)

II 95\% dei pazienti " $\mathrm{B}$ " ha raggiunto il target di adeguatezza dialitica con un miglioramento statisticamente significativo $(\mathrm{P}<0.0001) \mathrm{Kt} / \mathrm{V}$ da 1.03 (T0) a Kt/V 1.75 (T1).

Conclusioni: Il programma di miglioramento continuo implementato attraverso il coinvolgimento formativo da parte del personale infermieristico ai pazienti ha consentito di raggiungere il target prefissato per l'aumento sia del tempo di dialisi che del Kt/V.

021

\section{GARANTIRE UN'ASSISTENZA DI ALTA QUALITÀ ATTRAVERSO UNA VALUTAZIONE MULTIDISCIPLINARE BASATA SULLA COMPLESSITÀ DEL PAZIENTE}

Capoferri R. ${ }^{1}$, Poloni T. ${ }^{2}$, Moretti M. ${ }^{1}$, Pizzo A. ${ }^{1}$, Pelliccia F. ${ }^{3}$, Parisotto M.T. ${ }^{3}$ ${ }^{1}$ NephroCare Italia, Napoli, Italia; ${ }^{2}$ NephroCare Bolognini, Seriate (Bergamo), Italia; ${ }^{3}$ Fresenius Medical Care, NephroCare Coordination, Bad Homburg, Germania

Introduzione: I Nefrologi sono responsabili delle prescrizioni dei pazienti, tuttavia gli infermieri devono valutare, educare e svolgere tutti i compiti assistenziali legati ai pazienti in cura nel centro dialisi. Diversi studi hanno dimostrato differenze nella qualità della cura dei pazienti, offerta da infermieri meno esperti, rispetto a infermieri più esperti. Un'ulteriore difficoltà consiste nella variabilità dell'organizzazione del centro dialisi. Lo scopo di questo studio è stato quello di garantire gli stessi livelli di cura per i pazienti presenti nelle unità ospedaliere e per quelli nelle dialisi satellitari, considerando l'esperienza degli infermieri e la complessità del paziente.

Metodologia: Il livello di indipendenza dei pazienti è stato valutato attraverso il Modified Barthel Index (MBI) e le comorbidità sono state valutate con l'Age adjusted Charlson Comorbidity Index (AaCCl), per ottenere, così, lo status delle condizioni generali del paziente, basato sulla complessità dell'individuo. L'analisi è stata eseguita in un'unità di dialisi ospedaliera e in cinque unità satellite. Un totale di 180 pazienti è stato valutato da 48 infermieri.

Risultati: La valutazione degli indici $\mathrm{MBI}$ e $\mathrm{AaCCl}$ ha mostrato che i pazienti in tutte le unità di dialisi sono classificati tra minimamente complessi e complessi. II tempo medio di esperienza degli infermieri in dialisi era di $13.4 \pm 8$ anni.

Conclusioni: La valutazione dei pazienti ha rivelato che, indipendentemente dal tipo di unità di dialisi, non vi sono differenze significative tra i livelli di complessità dei pazienti. Il personale infermieristico dovrebbe, quindi, essere assegnato in maniera bilanciata sulla base del proprio grado di esperienza, nelle diverse realtà dei centri dialisi. La qualità delle cure dipende in gran parte da personale ben addestrato e da una valida organizzazione. 
033

PROGETTO DI EDUCAZIONE E FORMAZIONE PER LA CREAZIONE DI UN PDTA TRA NEFROLOGIA E TERAPIA INTENSIVA

Cardinali S., Verdacchi $R$.

SC Nefrologia e Dialisi, Azienda Ospedaliera di Perugia

Introduzione: L'aumento, nell'ultimo decennio, del numero di trattamenti dialitici per insufficienza renale acuta eseguiti al letto del malato nei vari reparti tra cui quelli intensivi ha portato l'azienda ospedaliera di Perugia a creare una procedura operativa per definire funzioni e responsabilità nella gestione multidisciplinare delle CRRT su pazienti critici. In particolare, sono stati individuati come reparti intensivi ad alta intensità assistenziale le rianimazioni (due nel nostro ospedale) e la terapia intensiva post cardiochirurgica. Il programma è stato acquisito dall'azienda ospedaliera, che ha anche provveduto a dotare di punteggio ECM il programma formativo.

Metodologia: Il lavoro è stato effettuato sotto l'egida e la partecipazione della direzione sanitaria e della medicina legale, che ne garantiscono sia la fattibilità che la liceità. Ha comportato la collaborazione delle equipe infermieristiche e mediche dei reparti interessati. II nostro gruppo di lavoro ha fornito strumenti e materiali per la formazione e l'addestramento del personale intensivista, con l'obiettivo di raggiungere le adeguate competenze richieste: lezioni frontali, simulazioni, presentazione di materiale didattico e retraining formativo on the job. Si è provveduto a definire la matrice delle responsabilità e delle competenze. Alle terapie intensive spetta il cambio sacca e il sistema di sorveglianza e monitoraggio del trattamento. All'equipe nefrologica spettano il compito di inizio e fine trattamento, il cambio delle impostazioni, il monitoraggio del trattamento e l'intervento su chiamata. Ai medici nefrologi spettano l'impostazione della terapia e qualunque intervento di cambio dello schema di terapia.

Risultati: Tutto il personale infermieristico intensivista ha raggiunto gli obiettivi richiesti dal progetto con un aumento della compliance collaborativa. I trattamenti in terapia intensiva sono ben organizzati e non ci sono né incomprensioni né sovrapposizioni di competenze e attività. I protocolli sono diventati protocolli aziendali a cui attenersi. I punti ECM sono stati ottenuti da tutto il personale. Il rate di assenza è stato modestissimo.

Conclusioni: Lo sviluppo e l'adozione di adatti PDTA concordati tra reparti e con la direzione sanitaria sono ottimi sistemi per regolare attività e competenze multidisciplinari. Le macchine da dialisi, i trattamenti dialitici e il personale medico e infermieristico, se non adeguatamente integrati, rappresentano dei semplici attori che, per collaborare al meglio e in modo armonioso, possono usufruire di adatti percorsi di formazione, di piani di lavoro condivisi e di risorse dedicate. In questo nostro progetto formativo abbiamo dimostrato che, mettendo insieme tutti questi strumenti, si ottengono risultati inattesi.

\section{EDUCAZIONE E FORMAZIONE}

\section{4}

M.R.C. E CONSAPEVOLEZZA: UN REPORT DELL'AMBULATORIO MaReA Bauducco M. ${ }^{1}$, Cerri $C^{2}{ }^{2}$, Menusan A. ${ }^{1}$, Soragna ${ }^{3}{ }^{3}$, Vitale $C{ }^{4}$

${ }^{1}$ CPSI A.S.O. Mauriziano, Torino; ${ }^{2}$ CPSE A.S.O. Mauriziano, Torino; ${ }^{3}$ Nefrologo A.S.O. Mauriziano, Torino; ${ }^{4}$ Direttore U.O. Nefro-dialisi A.S.O. Mauriziano, Torino

Razionale: Nella M.R.C. è fondamentale associare i momenti del curare a quelli dell'educare, per consentire alla persona con M.R.C. di aumentare le conoscenze della propria patologia.

Casistica e metodi: L'obiettivo dello studio è la valutazione dell'efficacia del programma educativo proposto nell'ambulatorio di M.R.C. avanzata, riorganizzato come da D.R. MaReA. È stato strutturato un questionario con risposte a scelta multipla per valutare il grado di conoscenza dei pazienti su M.R., dialisi e trapianto e somministrato ai 282 pazienti con Gfr. $<30 \mathrm{~mL} / \mathrm{m}$, seguiti presso il nostro ambulatorio.

Risultati: Dai dati preliminari emerge una non completa consapevolezza da parte di pazienti che da tempo sono seguiti presso il nostro ambulatorio: solo un terzo degli intervistati è a conoscenza di quale sia la funzione dei reni e un $30 \%$ di essi pensa che il non avere sintomi indichi l'assenza di M.R. e che il dolore debba essere necessariamente presente. Per la dialisi, solo il $60 \%$ afferma di avere informazioni e addirittura solo il $40 \%$ è a conoscenza che tale trattamento possa svolgersi anche a domicilio. Il trapianto di rene è considerato da un terzo dei pazienti un trattamento riservato esclusivamente alle persone più giovani e più dell' $80 \%$, è convinto che i reni "malati" debbano essere asportati per fare spazio a quelli trapiantati.

Conclusioni: Dall'analisi dei dati risulta fondamentale implementare programm informativi, chiari, completi e adeguati al grado di comprensione dei pazienti, al fine di fornire loro strumenti necessari per effettuare una scelta consapevole.

004

\section{ANALISI DELLE CONOSCENZE DIALITICHE DEL PAZIENTE IN} EMODIALISI (HD)

Petriello A., Leopaldi D., d'Aloya G., Fumagalli F., Limido A.

S.C. Nefrologia e Dialisi, ASST Fatebenefratelli Sacco, Milano

Introduzione: È noto che l'educazione in fase pre-HD migliora la qualità della vita e che riduce i costi sanitari e che il metodo faccia a faccia è più efficace della formazione tramite opuscolo. È, comunque, importante una costante verifica del corretto recepimento delle informazioni fornite.

Metodi: In 50 pazienti (42 M-8 F) con età mediana 66.5 anni (range 32-86) in HD da 2 a 240 mesi (media 52.7) è stato somministrato un questionario per analizzare il livello di conoscenza su dialisi, accesso vascolare e alimentazione. I dati sono stati analizzati in forma aggregata tramite Excel/Access.

Risultati: II $78 \%$ dei pazienti conosce a cosa serve I'HD, I' $84 \%$ cos'è la FAV, I'88\% gli alimenti che contengono elevate quantità di $\mathrm{K}$, il $20 \%$ quelli a elevato contenuto di P, il $72 \%$ gli effetti e il $66 \%$ l'apporto corretto di acqua giornaliera. La consapevolezza del sovraccarico idrico è direttamente proporzionale all'età dialitica.

Conclusioni: I nostri dati confermano che l'educazione al paziente con IRC stadio $5^{\circ}$ deve continuare dopo l'inizio dell'HD. L'informazione durante le sedute di HD può migliorare il controllo di $\mathrm{K}, \mathrm{P}$ e acqua. Il questionario si è dimostrato uno strumento idoneo per valutare i pazienti e colmare eventuali carenze, rafforzandone le conoscenze.

007

HEALTH CARE TELEPHONE COACHING PER PAZIENTI IN DIALISI PERITONEALE: UN'ESPERIENZA

Fadelli J., Manias E.P., Schinella D., Polesel J.

Azienda Ospedaliera S. Maria degli Angeli, Pordenone

Introduzione: Un piano di educazione sanitaria basato sull'health care telephone coaching (educazione sanitaria telefonica) e centrato sulla persona con malattia cronica porta a risultati positivi. Questi riguardano il mantenimento del self-care, dello stato di salute, della qualità di vita e della compliance e la riduzione dei ricoveri e delle spese mediche. Lo scopo dello studio è di descrivere la percezione di utilità del servizio health care telephone coaching da parte dei pazienti in dialisi peritoneale afferenti alla Struttura Operativa Semplice di Dialisi Peritoneale, ospedale di Pordenone - Azienda per l'Assistenza Sanitaria n. 5 "Friuli Occidentale", dove è già attivo il servizio "Telefono amico", rispetto a compliance, sicurezza e qualità di vita e di indagare i motivi che hanno spinto la persona a usufruire del servizio.

Metodologia: Studio osservazionale che coinvolge 38 persone, in dialisi peritoneale, che usufruiscono del servizio "Telefono amico" e afferenti alla S.O.S. di Dialisi Peritoneale, Ospedale di Pordenone, A.A.S.5. "Friuli Occidentale". Per la raccolta dati è stato somministrato un questionario autoprodotto con 22 item che indagano la percezione di self-care, sicurezza e qualità di vita in relazione al proprio vissuto e al servizio di health care telephone coaching che viene offerto. Il questionario è stato distribuito e raccolto nel periodo dal 1 Settembre al 23 Ottobre 2015.

Risultati: Il campione è composto da 38 persone, in cui il 71.05\% (27/38) è rappresentato da maschi e il 28.95\% (11/38) da femmine. L'età media è di 62.79 anni (range 27-81 anni, mediana 65.5 anni). II 100\% (38/38) del campione usufruisce del servizio "Telefono amico". Al quesito sul tempo di malattia rispondono 35/38 persone che soffrono di insufficienza renale cronica da un periodo che va da 9 mesi a 50 anni, con una media di 12.86 anni e una mediana di 10 anni. Richiedono aiuto con una media mensile di 2.05 volte. Il colloquio con l'infermiere durante la telefonata è riuscito a risolvere il problema nel $100 \%(38 / 38)$ dei casi e tutti i pazienti $(38 / 38)$ sono rimasti soddisfatti: grado complessivo uguale a 9.45 (range 8-10).

Conclusioni: Dall'analisi dei risultati sembrerebbe che il servizio "Telefono amico" riesca ad aumentare la percezione di sicurezza, compliance e qualità 
di vita della persona. I limiti di questo studio sono la numerosità numerica ridotta del campione e l'imperfezione del questionario autoprodotto; sarebbe utile modificare e validare il questionario per poi utilizzarlo per uno studio multicentrico.

\section{8}

PERCORSO FORMATIVO DI INSERIMENTO DELL'INFERMIERE NEOASSEGNATO IN DIALISI

Del Pioluogo $M$

UOC Dialisi, A.A.S.5, Pordenone

Introduzione: Negli ultimi anni, la professione infermieristica ha visto un susseguirsi di cambiamenti di tipo organizzativo, formativo e tecnologico, ai quali si aggiungono una costante carenza di organico e un rapido turnover del personale. II progetto nasce dall'esigenza di fornire un valido strumento di lavoro al personale che si appresta a intraprendere un nuovo percorso lavorativo, favorendo una buona formazione sul campo e un corretto sviluppo delle competenze infermieristiche, sia in termini di sicurezza e salute dei pazienti che di soddisfazione professionale.

Metodologia: attraverso un'analisi della letteratura, si sono approfonditi alcuni aspetti focali dell'andragogia e le evidenze scientifiche per l'elaborazione di un programma di apprendimento strutturato e codificato in grado di offrire un valido aiuto al discente, all'infermiere formatore e agli altri membri dell'equipe, garantendo la formazione del personale neo assegnato secondo standard condivisi, sia in termini di conoscenze specifiche da acquisire che di tempi adeguati al raggiungimento di livelli di autonomia operativa, compatibili con le necessità assistenziali della struttura.

Risultati: È stato elaborato un programma di apprendimento che consente al discente di essere attivamente partecipe nel processo formativo, attraverso I'acquisizione di abilità specifiche codificate, condivise dal gruppo e ordinate per grado di difficoltà. Prevede, infatti, una scheda, che analizza i singoli obiettivi (obiettivi specifici), fornisce spunti operativi (azioni) ed esplicita tempi di apprendimento (periodo formativo) e modalità di autovalutazione (indicatori di verifica), come occasione di condivisione con il mentor dei livelli raggiunti. La finalità è, quindi, quella di mettere il neo assegnato (NA) in condizioni di acquisire abilità e competenze per gestire in autonomia la seduta dialitica, dall'accoglimento del paziente al suo rientro a casa.

Il percorso si struttura in almeno sei mesi di affiancamento a un mentor, ma può essere modificato sulla base della turnistica, delle esigenze del NA e delle criticità affrontate in tale periodo, arrivando a un tempo formativo di massimo otto mesi.

Conclusioni: II programma, elaborato nell'Agosto del 2010, è stato utilizzato nell'inserimento di dodici infermieri neoassegnati e si è dimostrato un valido strumento di formazione sul campo e un modello formativo condiviso fra i mentor e gli altri membri dell'equipe.

Si può verosimilmente ipotizzare che questo percorso formativo possa e debba subire frequenti revisioni, sulla base delle nuove evidenze scientifiche e delle diverse esigenze operative.

\section{ACCESSI VASCOLARI E GESTIONE DELLE INFEZIONI NELLA PRATICA CLINICA}

005

\section{CRRT, ASPETTI TEORICI E PRATICI: L'EMODIAFILTRAZIONE "SLOW"}

Larini M.L. ${ }^{1}$, Greco M. ${ }^{1}$, Napoli M. ${ }^{2}$

${ }^{1}$ Infermiere, UOC Nefrologia e Dialisi, Asl Lecce, P.O. "S. Caterina Novella", Galatina, Lecce; ${ }^{2}$ Primario, UOC Nefrologia e Dialisi, Asl Lecce, P.O. "S. Caterina Novella", Galatina, Lecce

Introduzione: Agli albori della terapia dialitica, il trattamento sostitutivo renale fu visto come un mezzo per sostenere temporaneamente il paziente con IRA finché la funzione renale non riprendesse e non ritornasse, successivamente, nella norma. Con l'apertura di numerosi reparti di cardiochirurgia e con l'avvento di chirurgie sempre più invasive, il bisogno di terapie sostitutive renali più mirate e tollerate meglio dal malato clinico si fa sentire in modo crescente. Scopo del presente lavoro è di dimostrare l'efficacia di un modello di CRRT semplificato. Metodologia e risultati: L'emodiafiltrazione "slow": il modello 15 litri in 10 ore.
1) Esemplificare il protocollo dialitico (opzioni vari tipi di sacche; QB stabile 150 $\mathrm{mL} / \mathrm{m}$; QD $750 \mathrm{~mL} / \mathrm{h}$; post diluizione $750 \mathrm{~mL} / \mathrm{h}$; eparinizzazione continua da 250 a 750 u/h). 2) Ridurre i materiali di consumo. 3) Ottenere adeguate UF anche in pazienti con instabilità emodinamica (in 30 pazienti iperidratati abbiamo ottenuto, in un periodo medio di trattamento di 7-10 giorni, un decremento ponderale medio di circa $13 \mathrm{~kg}$, senza problemi di instabilità emodinamica; in nessun caso, il trattamento è stato sospeso per instabilità emodinamica; non è registrato alcun intervento per il sostegno della pressione in corso di trattamento; in un $5 \%$ di pazienti, il circolo era sostenuto da amine in atto già prima del trattamento). 4) Ottimizzare l'organizzazione dell'attività dialitica. 5) Fornire una dose dialitica adeguata: I'urea nel dialisato aumenta progressivamente la concentrazione durante il passaggio nel filtro fino a raggiungere quella plasmatica. Inutile avere flussi alti.

Conclusioni: Dei 2611 trattamenti eseguiti, 2083 sono stati effettuati in degenza nefrologica (circa l'80\%) e 528 in altri reparti. Negli ultimi 4 anni abbiamo trattato 404 pazienti affetti da IRA; 79 pazienti erano affetti da scompenso cardiaco (iperidratati), per un totale di 2611 trattamenti.

\section{9}

CONFRONTO FRA BTM + TWISTER VS TRANSONIC NELLA MISURAZIONE DELLA PORTATA DELLA FAV

Careja M. ${ }^{1}$, Cavallero $E^{1}{ }^{1}$, Forneris $G{ }^{2}{ }^{2}$, Vadori $M{ }^{2}{ }^{2}$, Roccatello $D^{2}{ }^{2}$

${ }^{1}$ Professionali Associati di Carmagnola, Torino; ${ }^{2}$ SCUD Nefrologia e Dialisi, Ospedale Giovanni Bosco, Torino

Introduzione: La misurazione della portata dell'accesso vascolare (Qa) è suggerita dalle LG per la sorveglianza della FAV. Abbiamo eseguito un lavoro di confronto della misurazione della portata del Qa con il BTM Fresenius + Twister (dispositivo che evita la disconnessione delle linee) vs Transonic HDM.

Metodologia: È stata valutata la portata della FAV in 12 pazienti (11 M $1 \mathrm{~F}$, età media 61.2 anni) durante lo stesso trattamento dialitico con le due metodiche. II BTM deriva Qa dal calcolo del ricircolo (comprensivo di quello cardiopolmonare, RCP), misurato a linee normali e invertite. La misurazione è stata eseguita da 2 a 6 volte per ogni paziente, per un totale di 63 coppie. Per la statistica, è stato utilizzato il software SPSS.

Risultati: Nessun ricircolo dell'accesso è stato registrato con Transonic $(=0 \%)$ come con $B T M(=9 \%$, tutto ascrivibile al RCP). La portata media osservata con BTM e Transonic è stata rispettivamente di $1359 \pm 759 \mathrm{~mL} / \mathrm{min}$ (mediana 1078) e di $1448 \pm 719 \mathrm{~mL} / \mathrm{min}$ (mediana 1240), con una correlazione di $r=0.86$.

Conclusioni: II BTM si conferma uno strumento affidabile e ripetibile nella misurazione della portata della FAV. Data la scarsa disponibilità del Transonic nei centri italiani, I'utilizzo del BTM con Twister può essere considerato una valida alternativa per la sorveglianza dell'accesso vascolare.

\section{9}

\section{IL TEAM DEGLI ACCESSI VASCOLARI}

Albertazzi D., Avaltroni O., Beltrandi R., Brintazzoli S., Di Marco T., Guadagno V., Magri Mazzini F.

Emodialisi Malpighi, Azienda Ospedaliera Sant'Orsola-Malpighi, Bologna

Introduzione: L'aumento dell'età dei pazienti uremici e la presenza di comorbidità che ne compromettono il patrimonio vascolare sono i motivi che hanno portato alla creazione del Team degli AA.VV.: il fine è la salvaguardia dell'accesso, requisito imprescindibile del trattamento dialitico.

Metodologia: Il team è composto e coordinato dall'infermiera responsabile degli accessi (RAV) e da altri colleghi che hanno fatto un percorso di sviluppo professionale e di acquisizione di competenze specifiche.

Gli strumenti di monitoraggio di I e II livello utilizzati sono: la cartella infermieristica informatizzata (che garantisce la continuità assistenziale), un ecografo, un monitor HDO3 per misurare la portata e il ricircolo dell'accesso vascolare, un fonendoscopio elettronico e il calcolo dell'efficacia dialitica.

Dal confezionamento della FAV in poi iniziano tutte le attività di controllo effettuate dal gruppo. Le criticità rilevate sono, poi, confrontate per decidere il percorso da intraprendere.

Risultati: Nel 2009, prima della costituzione del team, le FAV chiuse sono state 14 , di cui 10 per trombosi acuta.

Nel 2015, sono state documentate una media di 1.6/gg misurazioni della portata. Sono state eseguite 20 PTA su indicazioni cliniche e funzionali, 10 PTA solo su base clinica e 2 fistolografie senza PTA, con soli 6 episodi di chiusure 
delle FAV: 5 per trombosi acuta e 1 chiusa in elezione. Vi è una migliore comprensione dei parametri clinici da monitorare.

Conclusioni: La nascita del team ha prodotto effetti positivi su tutte le figure coinvolte nel processo assistenziale: sul paziente e sul suo accesso vascolare (fondamentale per una buona qualità di vita e una buona omeostasi), sul personale infermieristico, sul personale medico e sul sistema sanitario, con un utilizzo mirato delle risorse.

\section{6}

\section{LO SVILUPPO DELLE COMPETENZE INFERMIERISTICHE NELL'USO} DELL'ECOGRAFIA PER GLI ACCESSI VASCOLARI IN DIALISI

Cavallero E. ${ }^{1}$, Careja M. ${ }^{1}$, Forneris G. $^{2}$, Roccatello $D .^{2}$

${ }^{1}$ Professionali Associati di Carmagnola, Torino; ${ }^{2}$ SCUD di Nefrologia e Dialisi, Ospedale Giovanni Bosco, Torino

Introduzione: Nella progettazione della FAV e nella diagnostica delle complicanze, l'ecografia rappresenta un tool irrinunciabile. Esperienze preliminari ne fanno intravedere un possibile utilizzo bedside da parte dell'infermiere. Metodologia: È stata rivista la letteratura sulle competenze infermieristiche e sull'uso degli ultrasuoni per gli accessi vascolari in dialisi, è stato ideato un questionario regionale sulla disponibilità e sulle risorse, è stato sentito il parere di esperti ed è stata valutata la fattibilità di un percorso di apprendimento del personale della nostra Divisione. Gli obiettivi sono stati: il settaggio per il modulo vascolare, la capacità dell'identificazione dei vasi in asse lungo e corto e principi e tecniche di punzione ecoguidata ed ecoassistita.

Risultati: Dalla letteratura e dal questionario è emerso come siano pochi i centri dialisi in cui gli infermieri abbiano seguito un percorso di formazione o dispongano di risorse per questa attività.

Nella nostra esperienza, l'acquisizione delle competenze di base necessarie per un utilizzo corretto dell'ecografo ha richiesto un tempo relativamente breve. Conclusioni: L'utilizzo dell'ecografo da parte dell'infermiere è una realtà percorribile attraverso un percorso di formazione mirato. Esso può fornire un importante ausilio nella riduzione delle complicanze e nell'allungamento della vita dell'accesso vascolare.

041

\section{IL PERCORSO DEL PAZIENTE AFFETTO DA IRC: DALLA VALUTAZIONE} ALLA GESTIONE DELLA FAV

Cavina S., De Tommaso T., Malizia D., Montemagno C., Molinari C., Giurdanella P.

Policlinico Sant'Orsola-Malpighi, Bologna

Introduzione: Il paziente che viene preso in carico dall'unità operativa Nefrologia Dialisi e Trapianto diretta dal Prof. La Manna del Policlinico S. Orsola Malpighi di Bologna affetto da IRC viene inserito all'interno di un percorso per il confezionamento della fistola artero venosa in collaborazione con la chirurgia vascolare.

Metodologia: La suddetta U.O. ha costruito un percorso strutturato e standardizzato che il paziente affetto da IRC segue dal momento in cui viene preso in carico dall'ambulatorio pre-dialisi fino alla gestione della FAV durante la vita dialitica della persona.

Il paziente seguito all'ambulatorio pre-dialisi è sottoposto a controlli e a visite periodiche in modo da monitorare la funzionalità renale e viene inserito nel percorso di confezionamento della FAV solo quando i valori della funzionalità renale raggiungono il valore limite.

Una volta confezionata la FAV, il paziente rimane in carico all'ambulatorio pre-dialisi fino al momento in cui le sue condizioni non comportino il trattamento sostitutivo; a quel punto, un infermiere di dialisi esperto viene chiamato a valutare sia la maturazione che l'effettiva pungibilità della FAV stessa. Risultati: Durante il tempo trascorso in dialisi, l'andamento dell'accesso vascolare viene monitorato quotidianamente. Per quanto riguarda la fistola "in uso" che manifesta problematiche, le azioni sono molteplici e vanno da una valutazione sul corretto posizionamento degli aghi fino alla rivalutazione da parte del chirurgo vascolare, passando per la valutazione ecografica eseguita da un nefrologo e da un infermiere con competenze in ambito ecografico. Conclusioni: Nasce, all'interno del nostro centro, un gruppo di infermieri esperti in ecografia con l'obiettivo di fornire un'assistenza infermieristica sempre più completa e con il mandato di implementare la tecnica ecografica nella pratica quotidiana degli infermieri.
001

MEDICAZIONI A CONFRONTO PER LA GESTIONE DEI CVC TUNNELLIZZATI: ESPERIENZA DI UN CENTRO DIALISI FIORENTINO Milazzo E., Bandor I.

Istituto Fiorentino di Cura e Assistenza, Centro Dialisi Ulivella, Firenze

Introduzione: II Catetere Venoso Centrale (CVC) è una modalità di accesso vascolare largamente utilizzata nei pazienti sottoposti a trattamento emodialitico nei quali non sia possibile, per vari motivi, allestire una fistola artero-venosa. Scopo di questo studio è quello di valutare l'incidenza delle infezioni correlate a CVC tunnellizzati in seguito all'utilizzo di una medicazione adsorbente $d$ captazione batterica (Exit Green) a base di Dialchilcarbamoilcloruro (DACC). Metodologia: Ai fini dello studio, è stato analizzato un campione di $17 \mathrm{pa}$ zienti $(9 \mathrm{M} / 8 \mathrm{~F})$ sottoposti a trattamento emodialitico mediante l'utilizzo d CVC tunnellizzati. Tali pazienti sono stati medicati durante l'anno 2014 unicamente con garza sterile e cerotto, mentre, nel corso del 2015, è stata utilizzata una medicazione adsorbente a base di DACC. I dati sono stati confrontati allo scopo di valutare la variazione dell'incidenza delle infezioni CVC correlate nei 2 anni presi in esame.

Risultati: Nel 2014, abbiamo riscontrato 11 infezioni CVC correlate, con un'incidenza pari a 2.52/1000 gg di catetere, 2 delle quali determinate da MRSA Nel 2015, sono state rilevate 5 infezioni CVC correlate, con un'incidenza pari a $0.92 / 1000$ gg di catetere e un solo caso di MRSA. Dall'analisi dei dati, è emersa una riduzione del $63.5 \%$ dell'incidenza annua delle infezioni CVC correlate. Conclusioni: L'utilizzo della medicazione Exit Green a base di DACC ha notevolmente ridotto l'incidenza annua di infezione dei CVC tunnellizzati nei pazienti in emodialisi.

012

\section{EXIT SITE, TIPOLOGIE CUTANEE DIFFERENTI: UNICA MEDICAZIONE} D'ARGENTO

Calore C. ${ }^{1}$, Stochino M.G. ${ }^{1}$, Gioachin M. ${ }^{1}$, Pariani B. ${ }^{1}$, Caretta E. ${ }^{1}$, Fumagalli $F^{2}$, Fanelli S. ${ }^{2}$, Limido A. ${ }^{2}$, Cortesi $P^{3}$, Mento $A^{3}{ }^{3}$, Vrenna $G^{3}{ }^{3}$, Marino $F^{3}{ }^{3}$, Colussi $G .{ }^{3}$ ${ }^{1}$ Nefrologia e Dialisi Ospedale di Gallarate, Varese; ${ }^{2}$ Nefrologia e Dialisi Ospedale Fatebenefratelli, Milano; ${ }^{3}$ Nefrologia e Dialisi Ospedale Niguarda, Milano

È stato condotto uno studio osservazionale in tre ospedali della Lombardia, Gallarate, Fatebenefratelli e Niguarda di Milano, relativo alla valutazione dell'infezione exit site di CVC.

Da Giugno 2013 a Novembre 2015 sono stati osservati 70 pazienti portatori di CVC, di cui 24 hanno presentato segni di infezioni exit site di vario grado trattate tutte con medicazioni a base di pellicola d'argento senza uso di antibiotici; è stata anche considerata la differente tipologia cutanea (Fototipi I e $\mathrm{VI})$ di pazienti di diverse etnie.

Si è dimostrato che, dopo poche applicazioni di medicazioni a base d'argento, c'è stata una risoluzione del quadro infettivo senza bisogno di ulteriori terapie e senza esiti di discromie cutanee. Si è evidenziata anche una riduzione dei costi farmaceutici.

\section{0}

\section{CASE STUDY: MEDICAZIONE ALL'ARGENTO NELLE FISTOLE CON}

\section{TECNICA BUTTONHOLE}

Paradisi F. ${ }^{1}$, Benedetto M.T. ${ }^{2}$

${ }^{1}$ Infermiere, Unità Operativa Nefrologia e Dialisi, AUSL Imola, Bologna; ${ }^{2}$ Coordinatore Infemieristico, Unità Operativa Nefrologia e Dialisi, AUSL Imola, Bologna

Introduzione: Le medicazioni all'argento con azione antimicrobica possono essere utilizzate come una barriera per i microorganismi nelle ferite ad alto rischio di infezione o re-infezione.

Metodologia: Attraverso un monitoraggio fotografico, abbiamo osservato l'evoluzione di alcune complicanze che si sono manifestate nella tecnica di venipuntura a occhiello nella FAV (Fistola AteroVenosa). Le criticità sono state trattate, fino alla loro completa risoluzione, mediante l'utilizzo di medicazioni all'argento (NOVASOVAN-ag EMODIAL) nei punti di infissione degli aghi al termine del trattamento dialitico.

Risultati: In tutti i Case Study osservati si è evidenziata un'efficacia della medicazione all'argento nella risoluzione di infezione (una settimana), escara e lesione del punto di inserzione dell'ago nella FAV (due settimane). In un caso di FAV con panniculite, l'applicazione di una medicazione all'argento e l'avvio 
alla preparazione del tunnel per la tecnica a occhiello hanno portato alla riduzione dell'edema, tale da rendere più agevole la venipuntura.

Conclusioni: Riteniamo che il NOVASOVAN-ag utilizzato nella tecnica di venipuntura a occhiello offra importanti vantaggi, come la riduzione della flogosi e la prevenzione della formazione dell'escara, rendendo più agevole la venipuntura successiva.

\section{6}

LA MEDICAZIONE SORBA VIEW 2000 NELLA GESTIONE DEGLI ACCESSI VASCOLARI PERIFERICI

Specchio C.,_Armiento A., De Cata G., Del Priore M., Di Mezza A., Gattulli M.A., Leggieri A., Consagro L.

Struttura Complessa di Nefrologia, Dialisi e Trapianto Renale, Azienda "OORR", Foggia

La cateterizzazione vascolare periferica (PIVC) è una manovra frequente nell'ospedalizzazione acuta, poiché i pazienti necessitano di una somministrazione e.v. di fluidi e di farmaci durante la degenza. Una delle principali complicanze della manovra è la flebite.

La medicazione Sorba View 2000 è un dispositivo a finestra impermeabile e trasparente e consiste in un film in poliuretano ad alta traspirabilità che isola da agenti esterni e protegge la cute. Si applica con facilità e i tempi di permanenza arrivano fino a 7 giorni. Uno studio italiano ha verificato l'efficacia e la tollerabilità delle medicazioni Sorba View 2000 nella gestione dei C.V.C. rispetto alla gestione standard. La permanenza della medicazione è apparsa coerente alle Linee Guida del CDC di Atlanta. Nella nostra esperienza, dal 01/04/15 al 31/12/15, abbiamo utilizzato il dispositivo Sorba View 2000 nella gestione delle PIVC in 270 pazienti ricoverati nei quali non abbiamo evidenziato episodi di flebite né lesioni cutanee. Considerati il basso costo della medicazione, la facilità di applicazione e i vantaggi evidenziati, noi riteniamo che il dispositivo descritto sia un valido ausilio nella gestione quotidiana anche delle PIVC.

\section{0}

MEDICAZIONE DEL CATETERE VENOSO CENTRALE IN EMODIALISI CON EXIT-PAD AG

Albertini M., Busato A.

Servizio di Emodialisi, Ospedale S.S. Giovanni e Paolo, Venezia

Introduzione: L'accesso vascolare di prima scelta per i pazienti in emodialisi è costituito dalla fistola artero-venosa (FAV). Quando l'allestimento della FAV è controindicato o di difficile realizzazione (sistema venoso insufficiente, stenosi, trombosi, ecc.), l'alternativa più comune è rappresentata dal catetere venoso centrale (CVC).

Rispetto alla FAV, questo tipo di accesso presenta un significativo aumento del rischio di infezioni con relativo incremento dei costi ospedalieri derivato dalla maggiore morbidità dei pazienti.

L'interruzione permanente della cute, dovuta alla presenza del catetere, facilita l'insorgenza di batteriemie, la cui frequenza, riportata in letteratura, varia da 1 a 5.5/1.000 giorni-catetere nei pazienti con CVC tunnellizzato e da 0.04 a $0.55 / 1.000$ giorni-paziente nei pazienti con FAV.

Metodologia: II miglior modo di evitare le infezioni consiste nel rinforzare tutte le misure di prevenzione disponibili attraverso un coinvolgimento consapevole e attivo dei pazienti e di tutti gli operatori, uniformando il lavoro di quest'ultimi; inoltre, ogni centro provvede a monitorare le complicanze catetere correlate, le terapie effettuate e gli outcome clinici.

Le strategie di gestione dei pazienti portatori di CVC, gli strumenti e le pratiche atte a ridurre le CRBSI sono definite dalle Linee Guida internazionali (CDC Atlanta, 2011) e dai protocolli approvati dai centri.

Il protocollo del centro di Venezia prevede l'uso di Jodopovidone $10 \%$ in sol. acquosa e ipoclorito di sodio allo 0,057 gr per la disinfezione della cute. Ipoclorito di sodio al 0,57 gr per la disinfezione dei tubatismi.

In un arco temporale di circa 6 mesi, fra la primavera e l'estate del 2015, abbiamo avuto in carico 10 pazienti portatori di CVC permanente. Nel periodo considerato, 4 pazienti hanno presentato infezioni persistenti e lesioni cutanee di grado 3 nella sede dell'exit site. Le infezioni sono state trattate con terapia antibiotica sistemica; per le lesioni della cute, oltre alla metodica standard (si sono evitati disinfettanti a base di iodio), si è testata l'Exit-Pad $\mathrm{Ag}$, medicazione antibatterica con retina antiaderente a base di argento da applicarsi nel punto di inserzione del CVC; questa medicazione, di cui si sono valutati gli effetti e i benefici, è stata precedentemente impiegata con buoni risultati da altri centri.

Risultati: Dopo circa 2 mesi dall'applicazione della prima medicazione (3 medicazioni settimanali per paziente), nei 4 pazienti si è riscontrata una remissione dell'infezione con la riduzione dello stato della cute dal grado 3 al grado $0 / 1$ nella sede dell'inserzione del catetere.

Di rimarchevole, abbiamo osservato che, rispetto ai trattamenti con il solo antibiotico sistemico, la medicazione Exit-Pad Ag ha stimolato il completo ripristino del trofismo cutaneo.

In seguito, dopo la sospensione del trattamento, 2 pazienti hanno ripresentato segni di irritazione e flogosi di grado 1 e 2 . Un intervento tempestivo con questa medicazione ha portato alla risoluzione delle lesioni cutanee dopo 6/7 applicazioni, senza che si rendesse necessaria la somministrazione di antibiotici.

Conclusioni: Exit-Pad Ag si è dimostrato un presidio di semplice impiego ed estremamente efficace nel trattamento dell'exit site dei pazienti portatori di CVC. Non sono stati riscontrati casi di allergia o di intolleranza al prodotto, che è stato utilizzato con esito positivo, sia per la prevenzione delle infezioni sia per stimolare la riparazione del tessuto cutaneo.

\section{MONITORAGGIO, IPERIDRATAZIONE E ASPETTI NUTRIZIONALI}

009

\section{VALUTAZIONE DEL RISCHIO NUTRIZIONALE NELL'END STAGE RENAL} DISEASE

Graziani L. ${ }^{1}$, Del Puglia S. ${ }^{2}$, Sabattani S. $^{3}$

${ }^{1}$ Infermiere Dialisi, Azienda USL Toscana Centro, Ospedale San Giovanni di Dio, Firenze; ${ }^{2}$ Infermiere DS Dialisi, Azienda USL Toscana Centro, Ospedale San Giovanni di Dio, Firenze; ${ }^{3}$ Coordinatore Infermieristico, Azienda USL Toscana Centro, Ospedale San Giovanni di Dio, Firenze

Il rischio nutrizionale è considerato una delle priorità dei professionisti della salute odierna. Le raccomandazioni contenute nelle Linee Guida internazionali concordano sulla necessità che il rischio nutrizionale sia periodicamente valutato e trattato. La Regione Toscana ha previsto la Best Practice per la valutazione del rischio nutrizionale, che comprende la costruzione di processi assistenziali appropriati e richiede una gestione organizzata e sistematica, compresi l'utilizzo di test di screening validati e condivisi, inseriti nella cartella clinica (Rif. DRG 135 del 25/02/2008) individuale, e la realizzazione di un percorso diagnostico-terapeutico nutrizionale in caso $\mathrm{di}$ positività allo screening.

Materiali e metodi: II progetto di miglioramento in corso nel Centro Dialisi OSGD ASF prevede un test di screening per tutti gli utenti in trattamento sostitutivo renale. II test individuato dal gruppo di lavoro è il Geriatric Nutritional Risk Index (GNRI) per l'attendibilità e la rapidità di esecuzione. È basato su pochi dati che, mediante un'elaborazione matematica, producono il valore di score, in base al quale viene individuato un grado di rischio. In seguito al grado individuato vengono attivati i percorsi previsti dal progetto. Risultati: In seguito ai risultati del test di screening è stato attivato il percorso per le persone a medio rischio con indagini più approfondite, mediante la valutazione dietologica e l'impedenziometria.

Conclusioni: È stato presentato un progetto formativo per infermieri di dialisi per l'acquisizione di competenze relative all'esecuzione di GNRI e impedenziometria.

023

RUOLO DELL'INFERMIERE NELLA GESTIONE E NEL MONITORAGGIO DELLO STATO DI IDRATAZIONE DEL PAZIENTE IN EMODIALISI

Menichelli L. ${ }^{1}$, Ordonez D. ${ }^{1}$, Trombia L. ${ }^{2}$, Pizzo A. ${ }^{2}$

${ }^{1}$ NephroCare Italia, Centro Dialisi NephroCare Cerlab, Roma; ${ }^{2}$ NephroCare Italia, Napoli

Introduzione: La malattia cardiovascolare (CVD) resta la causa principale d morte nei pazienti in terapia dialitica (HD). II raggiungimento di un normale stato di idratazione (HS) è uno dei maggiori target in HD, poiché tale alterazione è correlata a ipertensione arteriosa, ipotensioni intradialitiche, edemi polmonari e periferici, scompenso cardiaco e ipertrofia ventricolare sinistra. 
Lo scopo di questo studio è di creare un Fluid Management Program (FMP) atto a prevenire e a ridurre l'esposizione alla sovraidratazione dei pazienti in HD.

Metodologia: Lo studio è stato eseguito su 120 pazienti di un centro Dialisi di Roma, da Maggio a Novembre 2014. II FMP è stato supportato dal referente infermieristico attraverso il training al personale per la creazione, la revisione e il monitoraggio del FMP. La valutazione dell'HS è stata effettuata attraverso la misurazione spettroscopica della bioimpedenza della composizione corporea (BIS). Mensilmente sono state effettuate riunioni per la valutazione dei risultati dei pazienti a target e cioè con un rapporto di iperidratazione $(\mathrm{OH}) \mathrm{e}$ di acqua extracellulare (ECW) in pre-HD inferiore al 15\% (relative $\mathrm{OH}$ ). Risultati:

Maggio 2014: 53.90\%;

Giugno 2014: 55.14\%;

Luglio 2014: 72.92\%;

Agosto 2014: 78.70\%;

Settembre 2014: 68.20\%;

Ottobre 2014: 76.40\%;

Novembre 2014: 85.84\%.

Conclusioni: Al termine del nostro studio abbiamo ottenuto un aumento del 31.94\% dei pazienti a target. L'utilizzo di uno strumento validato attraverso il quale attuare una valutazione obiettiva dell'HS, combinato a una pianificazione e a una personalizzazione valide del FMP, consente di migliorare I'individuazione del peso secco e di ridurre le complicanze dei pazienti in HD, senza costi aggiuntivi.

013

VALUTAZIONE DEL GRADO DI IDRATAZIONE DA PARTE DEL PERSONALE INFERMIERISTICO IN UN AMBULATORIO DI PRE-DIALISI

Federici G.

UOS di Dialisi Peritoneale, Ospedale C. e G. Mazzoni, Ascoli Piceno

Introduzione: L'iperidratazione è caratterizzata da un'aumentata quantità e da un'alterata distribuzione dei liquidi corporei. I pazienti (pazienti) iperidratati in insufficienza renale presentano un rischio di mortalità aumentato rispetto ai normoidratati.

Metodologia: Nel nostro Ambulatorio di Pre-dialisi, sono valutati, dal punto di vista clinico-laboratoristico, pazienti allo stadio $4^{\circ}$ e allo stadio $5^{\circ} \mathrm{di}$ IRC (ossia con eGFR di 30-15 mL/min e $<15 \mathrm{~mL} / \mathrm{min}$, rispettivamente).

L'Ambulatorio ha una cadenza monosettimanale ed è gestito dal Nefrologo della sezione di Peritoneodialisi e da un'Infermiera della stessa sezione che, ad ogni visita, determina i parametri come: Peso (PS) e Pressione Arteriosa (PA) clino e orto.

I pazienti al $4^{\circ}$ stadio di IRC vengono visitati con cadenza bimensile, mentre i pazienti al $5^{\circ}$ stadio di IRC hanno necessità di controlli mensili.

Nel momento in cui, all'esame fisico, eseguito dal medico, si rileva un incremento ponderale, possiamo disporre di ulteriori test diagnostici non invasivi, come la Bioimpedenza, a cui, recentemente, si è aggiunto l'esame ecografico (ECT), che ci permettono di percepire le precoci variazioni dello stato idrico, coadiuvando, così, efficacemente l'azione medica tempestiva. Tale esame ecografico si è reso disponibile dopo che la nostra UOC di Nefrologia e Dialisi ha organizzato un corso teorico-pratico di ecografia, con ECM, per il personale infermieristico, con l'obiettivo di rendere accessibile una rapida valutazione del polmone e della vena cava inferiore ( $\mathrm{VCl}$ ) e il controllo degli accessi vascolari per emodialisi.

Pertanto, dopo un periodo di addestramento, sotto la guida di un nefrologo-ecografista, il corso ci ha consentito di valutare le condizioni di polmone "umido" o "asciutto" e della $\mathrm{VCl}$, relativamente al suo diametro durante le escursioni respiratorie.

Dal punto di vista tecnico, l'esame ecografico prevede una scansione trasversale sottocostale $\mathrm{dx}$ per la $\mathrm{VCl}$ e del distretto toracico posteriore per il polmone. Nel primo caso, si hanno utili indicazioni sulla capacità di collabimento dei vasi durante la dinamica respiratoria, mentre, nel secondo, in presenza di un versamento pleurico tra i due foglietti, si osserva uno scollamento (anecogenicità) dovuto al liquido pleurico.

Risultati: Abbiamo valutato routinariamente gli 11 pazienti al $5^{\circ}$ stadio di IRC, con maggior rischio di morbidità. Abbiamo avuto una riduzione dei ricoveri per sovraccarico idrico e abbiamo migliorato il self-care del paziente. Conclusioni: La non invasività dell'ECT, la sua ripetibilità e la possibilità di rispondere in termini di sìno al quesito clinico e, in tal caso, alle variazioni del diametro della $\mathrm{VCl}$ e del versamento pleurico rappresentano un valido aiuto nella conduzione di quei pazienti che necessitano di uno stretto controllo clinico.

037

RUOLO DELLA NUTRIZIONE PARENTERALE INTRADIALITICA (IDPN) E SUE IMPLICAZIONI CLINICHE: LA NOSTRA ESPERIENZA

Rossitto G. ${ }^{1}$, Vittorio A. ${ }^{2}$, Floridia $S^{2}{ }^{2}$, Gianni . $^{2}$, Denaro $G .^{2}$, Veneziano $D .{ }^{2}$, Marraro L. ${ }^{2}$, Maggio $C{ }^{2}$, Bongiovanni N. ${ }^{1}$, Zappulla $C .{ }^{1}$, Vita A. ${ }^{1}$, Sessa $C^{2}{ }^{2}$, Reina $A .{ }^{1}$ Di Franco S. ${ }^{2}$, De Luca M. ${ }^{2}$, Corsaro L. ${ }^{2}$, Gulino D. ${ }^{1}$, Bascetta D. ${ }^{1}$, Gianni S. ${ }^{2}$

${ }^{1}$ Ambulatorio di Emodialisi Floridiano, Floridia, Siracusa; ${ }^{2}$ Servizio Nefrodialitico Tike, Siracusa

Il problema della malnutrizione in dialisi rappresenta un fenomeno clinico d primaria importanza, predittivo di morbidità e mortalità. La patogenesi della malnutrizione negli emodializzati è multifattoriale e riconducibile all'inadeguata assunzione di calorie e proteine e alla perdita di nutrienti durante il trattamento in pazienti già "fragili". In tale contesto, risulta fondamentale i ruolo dell' "infermiere di continuità", che intercetta per primo i segnali dello squilibrio tra fabbisogno/introito e gli scompensi iniziali dell'organismo de paziente.

Alla luce di tale consapevolezza, tra i pazienti emodializzati afferenti a due ambulatori, sono stati arruolati 10 soggetti a rischio di malnutrizione di età compresa tra i 65 e gli 85 anni, caratterizzati da linfopenia, creatininemia $<8 \mathrm{mg} / \mathrm{dL}$, albuminemia $<3 \mathrm{~g} / \mathrm{dL}$ e $\mathrm{BMI}<18$ e che presentavano, all'anamnesi alimentare, un introito giornaliero $<20 \mathrm{kcal} / \mathrm{kg}$ corporeo. Abbiamo, dunque, trattato il campione per 12 sedute con soluzione per IDPN composta da emulsione lipidica e soluzioni di glucosio e amminoacidi, impostando come durata del trattamento, 240 minuti e velocità di infusione $150 \mathrm{~mL} / \mathrm{min}$ (raggiunta gradualmente) e conteggiando il volume di infusione $(625 \mathrm{~mL}$ ) nel calo peso totale. Al termine del periodo di trattamento, alla rivalutazione $\mathrm{s}$ è dimostrato l'incremento statisticamente significativo $(\mathrm{P}<0.05$, Analisi della Varianza) del peso, del BMI e dei parametri biochimici (creatininemia, linfociti, albumina). Anche la valutazione dello stato di benessere, ottenuta prima e dopo il trattamento somministrando un questionario, ha dimostrato un significativo miglioramento soggettivo. Sulla base delle nostre osservazioni, I'IDPN sembra migliorare i parametri nutrizionali, risultando utile quando le altre strategie nutrizionali non sono disponibili, pur suggerendo poco sulla sopravvivenza.

\section{LA TECNICA ENERGETICA DEL THERAPEUTIC TOUCH}

003

\section{LE TERAPIE COMPLEMENTARI IN NEFROLOGIA}

Carrossa F. ${ }^{1}$, Bovo L. ${ }^{2}$, Burrai $F^{3}$

${ }^{1}$ Istituto Oncologico Veneto IOV, IRCSS, Padova; ${ }^{2}$ Infermiera Libera Professionista, Padova; ${ }^{3}$ Azienda Ospedaliero Universitaria di Bologna, Bologna

Introduzione: Le terapie complementari (TC) sono un insieme di interventi olistici che presentano evidenze scientifiche e che si integrano in maniera sinergica e complementare con l'assistenza convenzionale. Le TC, negli ultim anni, hanno mostrato un notevole incremento nel loro uso nella popolazione italiana e mondiale. Le TC maggiormente usate in nefrologia sono la musicoterapia, l'omeopatia, l'agopuntura, l'aromaterapia, la guided imagery, la preghiera, la fitoterapia, il massaggio, le tecniche di respirazione, la meditazione, lo yoga, il rilassamento e le tecniche energetiche.

Scopo: Individuare, valutare e sintetizzare l'efficacia delle TC in nefrologia.

Metodi: È stata eseguita una revisione sistematica delle letteratura di studi RCT utilizzando la metodologia PRISMA, consultando 6 banche dati e includendo solo RCT in lingua inglese, full text.

Risultati: Gli RCT individuati sono 18 , con le seguenti significatività: riduzione dell'ansia con la musicoterapia, miglioramento del sonno, del prurito e della qualità di vita con l'agopuntura, miglioramento della vitalità mentale e fisiologica con la moxibustione, riduzione del dolore con l'aromaterapia, riduzione della proteinuria con la fitoterapia, miglioramento del sonno con le tecniche di rilassamento e guided imagery, miglioramento della qualità del sonno, della fatigue, dei valori di urea, del numero degli eritrociti, de 
colesterolo e della creatinina con lo yoga e miglioramento della qualità di vita con tecniche bioenergetiche. I pazienti hanno mostrato un'estrema ricettività nell'uso delle TC.

Conclusioni: Alcune TC mostrano evidenze per il loro utilizzo nella pratica assistenziale nefrologica, ma, in generale, presentano limiti di tipo metodologico, come la bassa numerosità campionaria e la potenza dello studio. Nella ricerca sono necessari un incremento degli studi RCT, un aumento della dimensione campionaria e una formazione infermieristica specifica nelle TC.

\section{IL TRAINING IN DIALISI PERITONEALE: PASSATO, PRESENTE E FUTURO TRA STANDARDIZZAZIONE E PERSONALIZZAZIONE. MODELLI A CONFRONTO: EMODIALISI VS DIALISI PERITONEALE}

\section{2}

TRAINING AI PAZIENTI PORTATORI DI CATETERE PERITONEALE PER LA VALUTAZIONE DEI SEGNI E DEI SINTOMI PRECOCI DI INFEZIONE DELL'EXIT SITE

Enni P.M.

Dialisi Peritoneale Az. Ospedale S. Maria, Pordenone

Introduzione: La prevenzione e la cura delle infezioni del punto di uscita del catetere peritoneale (exit site) sono considerate da tutti gli Autori delle Linee Guida essenziali per il mantenimento efficace della dialisi peritoneale stessa. È importante rilevare che le strategie per prevenire e ridurre il rischio di infezione comportano la cura rigorosa dell'exit site e un'attenzione meticolosa nel saper riconoscere segni precoci di infezione.

Obiettivi: Uniformare l'interpretazione dei segni flogistici degli exit site e condividere la metodologia semplificandone la lettura per permettere un intervento precoce atto a evitare le complicanze di infezione che potrebbero insorgere.

Metodologia: Una revisione della letteratura ha evidenziato che le peritoniti dovute a un'infezione del punto d'uscita del catetere peritoneale si possono verificare in oltre il $20 \%$ dei pazienti sottoposti a dialisi peritoneale. Nella nostra Unità Operativa cinque anni fa, durante la stesura dei protocolli e delle procedure che disciplinavano la dialisi peritoneale, abbiamo cercato di preparare anche una tabella per il riconoscimento e la classificazione dei segni e dei sintomi che accompagnavano l'osservazione e la cura dell'exit site. Molto importante è stato condividere tra tutto il personale che si occupava del training dei pazienti che arrivavano alla nostra attenzione quali erano le raccomandazioni da consegnare alle persone addestrate al momento dell'invio a domicilio. I report sulla cura del punto d'uscita erano focalizzati specificatamente sull'identificazione e sulla valutazione secondo scala criteri di Twardowski modificata e venivano condiviso tra il personale avvalendosi anche di un registro informatizzato dove annotare gli episodi infettivi. In tempi più recenti, venivano registrate in cartelle informatiche, nominate per ogni paziente, tutte le immagini fotografiche eseguite o dall'equipe o dall'assistito stesso al suo domicilio e successivamente inviate al team e da esso visionate. Risultati: Dalla nostra esperienza attraverso la raccolta dati, abbiamo identificato quali erano i segni e i sintomi precoci di infezione, condividendo una formula da trasferire ai pazienti in cura. La comparsa dei segni e i sintomi di GRADO 2 e/o secrezione accompagnato da fastidio o dolore, imponeva ai pazienti la premura di contattare telefonicamente il personale dedicato nel centro che li assisteva per organizzare una visita di controllo e le cure del caso.

Conclusioni: Rielaborando i dati raccolti in questi cinque anni di attività, possiamo affermare che la stesura e l'utilizzo della scala di Twardowski semplificata hanno aumentato nell'intera equipe la conoscenza delle complicanze precoci infettive dell'exit site e ci hanno permesso, nonostante gli episodi infettivi, di evitare le manovre di rimozione e di riposizionamento del catetere peritoneale per problematiche infettive non risolte. I nostri dati dimostrano che, nel 2011, con 28 pazienti, ci sono stati tre riposizionamenti di cateteri peritoneali per infezioni exit site non risolte, mentre, nell'anno 2015, con 45 pazienti in trattamento dialitico, nessuno ha subito manovre chirurgiche per le problematiche succitate. L'aumentata capacità di contribuire alla stesura di un programma di educazione terapeutica ha permesso di integrare nuove figure deputate alla cura del paziente (un'infermiera e un medico), senza compromettere i risultati ottenuti. In definitiva, ci sentiamo di affermare che rispettare le Linee Guida e condividerle ha migliorato di sicuro delle condizioni di vita dei pazienti, aumentando la loro autonomia e non trascurando il loro operare in sicurezza nel proprio domicilio.

016

MANGIARE GIUSTO CON GUSTO

Bianchi S.

Azienda USL Toscana Centro, Massarella Fucecchio, Firenze

Introduzione: La metodica dialitica non sempre riesce a rimuovere alcune sostanze largamente contenute in alcuni cibi. Seguire una dieta a basso contenuto di fosforo (priva di latte e derivati, uova e altri alimenti) vuol dire sacrificio e rinunce, soprattutto in relazione alle abitudini alimentari e allo stile di vita che spesso si conduce. Ecco l'idea di preparare un pranzo completo con alimenti scelti e cotti seguendo opportune procedure, così da far apprezzare la dieta corretta mangiando cibi giusti e gustosi dall'aperitivo al dolce.

Metodologia: Esecuzione di un pranzo con alimenti cucinati e preparati dal personale medico e infermieristico dell'ambulatorio di dialisi peritoneale presso una cucina attrezzata che ha permesso di eseguire e servire il pranzo a 70 persone (pazienti e familiari). Nel corso della spesa e della preparazione degli alimenti, sono stati eseguiti foto e filmati, con particolare attenzione al tipo di cibo comprato (margarina vegetale, latte di soia, albume d'uovo) e al tipo di cottura. Al termine del pranzo sono state proiettate immagin relative alla preparazione dei piatti del menù con una lezione di educazione nutrizionale-alimentare.

Risultati: Oltre a ottenere l'attenzione degli assistiti e del care giver nella preparazione degli alimenti, avendo assaggiato direttamente il cibo preparato, i giorno successivo sono venuti in ambulatorio per eseguire il controllo ematico e tutti gli assistiti presentavano valori del fosforo nei range desiderati (4-4.5 mg/dL).

Conclusioni: Abbiamo raggiunto l'obiettivo di dimostrare che un acquisto e una preparazione corretti del cibo possono consentire anche a un dializzato di non rinunciare al piacere della tavola.

\section{NUOVE FRONTIERE NEL TRAPIANTO DEL RENE}

027

CARTELLA INFERMIERISTICA RELATIVA AL PAZIENTE CANDIDATO AL TRAPIANTO DI RENE: ESPERIENZA DEL CENTRO TRAPIANTI DI TORINO Curci D., Dell'Olio R., Appendino I., Biancone L.

Unità Trapianti Renali, AOU Città della Salute e della Scienza di Torino, Presidio Molinette, Torino

Introduzione. Presso I'Unità Trapianti Renali dell'AOU Città della Salute e della Scienza di Torino, la documentazione necessaria per l'immissione in lista del candidato al trapianto renale riporta relazioni di molti specialisti che valutano l'idoneità del paziente al trapianto. L'idea di integrare tale documentazione con una cartella infermieristica è emersa durante un corso di formazione rivolto agli infermieri dei centri nefrologici piemontesi. In effetti, è l'infermiere del centro dialisi che ha il contatto più continuativo con il paziente e, grazie alla specificità del suo approccio, è in grado di descriverne i bisogni socio-assistenziali e il modello di aderenza terapeutica, requisiti fondamentale per un buon mantenimento del rene trapiantato.

Metodologia: È stato progettato un corso di formazione (2 edizioni) organizzato in gruppi di lavoro, ai quali hanno partecipato 30 infermieri coinvolt nel percorso del paziente candidato al trapianto di vari centri di Piemonte e Valle D'Aosta, per la realizzazione della cartella infermieristica. È seguita una sperimentazione di 6 mesi dove, per tutti i nuovi pazienti immessi in lista e per tutti i pazienti convocati per il trapianto, veniva richiesta la compilazione della cartella. Terminata la sperimentazione, tutti i centri sono stati contattati per valutare difficoltà incontrate e/o eventuali migliorie.

Risultati: Hanno recepito e attuato le indicazioni della sperimentazione 21 centri dialisi su 26 (81\%). Le cause di non adesione riferite sono di natura organizzativa. La cartella ha favorito la conoscenza del paziente e la comunicazione tra gli operatori coinvolti nel percorso del candidato al trapianto. Si è dimostrato anche un potenziale strumento di orientamento per il colloquio dello psicologo 
Conclusioni: La cartella infermieristica completa, così, la documentazione pre trapianto, permettendo di avere una valutazione clinico-assistenziale del candidato al trapianto.

\section{2 \\ DONATORE E TRAPIANTO RENALE: VALUTAZIONE DELLA QUALITÀ DI VITA DOPO LA DONAZIONE \\ De Natale R. ${ }^{1}$, Curci $D .^{2}$, Biancone L. ${ }^{2}$, Dimonte $V^{3}$ \\ ${ }^{1}$ Università degli Studi di Torino, Corso di laurea in Infermieristica, Torino; ${ }^{2}$ Dipartimento Trapianti, Nefrologia, Dialisi e Trapianto, Città della Salute e della Scienza di Torino, Torino; ${ }^{3}$ Dipartimento di scienze della Sanità pubbli- ca e Pediatriche, Torino}

Introduzione: Oggi il trapianto di rene rappresenta la terapia d'elezione per I'Insufficienza Renale Avanzata; in particolare, quello da donatore vivente garantisce una cospicua percentuale di effetti positivi sia per il ricevente che per il donatore. Ma la donazione da vivente è una condizione moralmente complessa: i donatori, infatti, vengono sottoposti a una serie di rischi fisici e psicologici, con possibili ripercussioni sulla loro QdV.

Metodologia: L'obiettivo dello studio è di sapere se i donatori beneficiano o meno di una QdV superiore rispetto alla popolazione generale dopo la donazione attraverso due questionari somministrati (tra Aprile e Agosto 2015) presso l'Ambulatorio Pre-Trapianto Reni del PO Molinette: PGWBI e SF-12. I risultati ottenuti sono, poi, stati confrontati con i dati della popolazione generale, al fine di poter comprendere possibili discordanze e differenze significative, usando come riferimento $\mathrm{p}<0.05$ (livello di significatività).

Risultati: Con il PGWBI emerge che i donatori della popolazione campione hanno una QdV significativamente superiore rispetto alla popolazione generale anche in seguito alla donazione $(p=0.01)$. In particolare, le donne del campione hanno ottenuto un risultato statisticamente superiore nella sfera "Positività e Benessere" rispetto alla popolazione femminile generale $(p=0.02)$; gli uomini hanno ottenuto risultati statisticamente migliori nelle sfere "Ansia, Depressione, Positività e Benessere" rispetto alla popolazione maschile generale ( $p=0.02-0.03-0.03)$.

Con il questionario SF12, invece, emergono differenze statisticamente significative per la dimensione psicologica $(p=0.004)$, ma non per quella fisica $(p=0.31)$; in particolare, sono le donne del campione a presentare un benessere mentale significativamente migliore rispetto alla popolazione generale $(p=0.004)$. Inoltre, i due soggetti con il più basso punteggio nella dimensione fisica hanno ottenuto il più alto punteggio nella dimensione psicologica. Conclusioni: Nel trapianto di rene, molto spesso l'attenzione è orientata più verso il ricevente che verso il donatore, perdendo di vista quest'ultimo in seguito alla donazione. PGWBI e SF-12 hanno permesso di scoprire possibili cambiamenti, sia nel corpo che nella mente, dovuti alla donazione. Sarebbe opportuno indagare sul benessere fisico e mentale già prima della donazione, per poter costruire e realizzare, in accordo con il donatore, un percorso individualizzato e mantenere il più elevato stato di salute in relazione a tale esperienza.

\section{5}

\section{UN CASO CLINICO DI BACILLO PIOCIANEO}

Bartiromo M., Bugianelli S., Rossi F., Rossi S., Minetti E.E.

Nefrologia Dialisi e Medicina del Trapianto, Azienda Ospedaliera Universitaria Careggi, Firenze

Introduzione: Le infezioni batteriche nel primo trimestre post trapianto d'organo solido sono molto frequenti. In modo particolare, sono molto frequenti le infezioni urinarie e quelle polmonari. Sotto il nome di Pseudomonas, definito anche bacillo piocianeo, è compreso un gran numero di batteri soprofiti, Gram-negativi e aerobi, che si trovano comunemente sul suolo, nelle acque e in molti altri ambienti. La specie Pseudomonas aeruginosa è quella che più spesso si ritrova nelle malattie dell'uomo, specialmente in quelle che interessano le parti più basse delle vie aeree. La Burkholderia (precedentemente indicata come Pseudomonas) cepacia è apparsa come un importante patogeno respiratorio nei soggetti immuno-compromessi e nei pazienti con Fibrosi Cistica (FC). Pseudomonas aeruginosa è un bacillo patogeno Gram-negativo a forma di bastoncino, aerobio stretto, che produce un pigmento fluorescente di colore azzurro (piocianina) o verde (pioverdina) o rosso scuro (piorubina o nero, piomelanina). Frequentemente presente su mucose indenni, si trova anche nell'ambiente per la notevole resistenza e le scarse esigenze nutrizio- nali. Ha una notevole resistenza agli antibiotici, soprattutto ai ceppi di origine ospedaliera.

Metodologia: Il protocollo di terapia immunosoppressiva utilizzato per i paziente ha previsto l'utilizzo di una terapia di induzione e di una terapia di mantenimento. La terapia di induzione è stata caratterizzata dal basiliximab + corticosteroide (nell'intra operatorio) e inibitore delle calcineurine (quest'ultimo nel post operatorio). La terapia di mantenimento ha previsto uno scalaggio del corticosteroide prima in vena e, poi, per via orale; l'inibitore delle calcineurine (sec. protocollo del centro); il basiliximab, un anticorpo monoclonale di tipo chimerico che si lega al recettore dell'interleuchina delle cellule $\mathrm{T}$ dato (basiliximab in prima dose di $20 \mathrm{mg}$ IV al tempo zero e dopo quattro giorni post trapianto); l'inibitore delle calcineurine, nello specifico la ciclosporina alla dose di $0.6 \mathrm{mg} / \mathrm{kg}$, divisa in due somministrazioni per via orale dopo l'uscita dalla sala; l'acido micofenolico antiproliferativo $360 \mathrm{mg} 2$ $\mathrm{cp} \times$ due dopo I'uscita dalla sala; il metilprednisolone $500 \mathrm{mg}$ IV in bolo in sala e, poi, a scalaggio nel post trapianto secondo un protocollo di centro; una terapia al rientro in degenza medica caratterizzata da una terapia antibiotica con sulfametoxazolo/trimetroprim $160 \mathrm{mg}+800 \mathrm{mg}$ 1/2 dose; un inibitore d pompa pantoprazolo $20 \mathrm{mg} /$ die; la nistatina lavaggi orali ( 3 x die); l'amlodipina $5 \mathrm{mg}$; l'eparina HPM secondo peso.

Risultati: Paziente maschio di 54 anni. ADPKD diagnosticata da 10 anni circa con insufficienza renale cronica progressiva dal 2013; ipertensione arteriosa in trattamento farmacologico con calcio-antagonista da circa dieci anni in terapia sostitutiva emodialitica dal 2014 e diuresi residua di circa un litro nelle 24 ore. Il paziente non è diabetico, non è cardiopatico e non ha allergie riferite né a farmaci né ad altro. II paziente non ha subito interventi chirurgici in anamnesi. II paziente risulta idoneo a essere sottoposto a trapianto ed entra in lista trapianto renale da donatore cadavere nel 2014 nel primo trimestre e senza avere in anamnesi episodi di infezioni urinarie degne di nota e con solamente due episodi di macroematuria. Nel primo trimestre del 2015 viene chiamato per essere sottoposto a trapianto di rene. II paziente in buona salute viene trapiantato con un rene sinistro in FID (DL $112 \mathrm{~cm}$ ) secondo un protocollo Basiliximab, CYC, MPA e CS (cfr. sezione metodologia). II trapianto presenta un buon successo; il rene trapiantato autonomizza la funzione con diuresi e depurazione già durante la prima settimana del post operatorio. I paziente, dal punto di vista generale, sta bene e viene dimesso in $18^{\text {a }}$ giornata. Nello schema terapeutico, oltre alla terapia IS secondo scali protocollari, un calcio-antagonista a basse dosi mezza compressa da $5 \mathrm{mg}$ di amlodipina un IP (pantoprazolo $20 \mathrm{mg}$ die; ranitidina $150 \mathrm{mg}$ x due; nistatina sec protocollo; sulfametoxazolo/trimetroprim $160 \mathrm{mg}+800 \mathrm{mg}$ 1/2 cp). La creatinina alla dimissione è pari a $1.6 \mathrm{mg} / \mathrm{dL}$ e il resto degli esami è nei range corretti. Gli indici infiammatori sono negativi con colture urinarie negative. II Doppler del rene trapiantato con IR indici resistenza nei range corretti. All'ecografia di controllo assenza di raccolte o dilatazioni e la sonda doppia J è in sede e verrà rimossa come da protocollo dopo la quarta settimana post trapianto. Dopo circa due settimane dalla dimissione, un rialzo della creatinina fa ricoverare il paziente per essere sottoposto a biopsia renale nel sospetto di segn di tossicità al CNI (CYC), visti i frequenti livelli elevati di Co e C2. Alla biopsia del rene (BRTx), non emergono segni di tossicità al CNI ma segni di infiltrato linfocellulare attivo. Si decide di sottoporre il paziente a un trattamento in vena con tre boli di CS e switch con tacrolimus al posto della ciclosporina. Alla dimissione, la creatinina è intorno a $1.8 / 1.9 \mathrm{mg} / \mathrm{dL}$. II paziente viene, quindi, dimesso e riaffidato all'ambulatorio del follow-up. Nei giorni che seguono, i valori di creatinina si mantengono stabili, ma l'iniziale ipersensibilità alla glicemia e vista durante l'ultimo ricovero induce la necessità di cominciare l'ipoglicemizzante orale a dosi stabili. Nelle settimane che seguono, assistiamo a sei episodi di infezione delle vie urinarie (IVU) con presenza di Pseudomonas aeruginosa nelle urine. Gli ultimi tre episodi infettivi complicati da sepsi grave costringono il paziente a due ricoveri in ambiente specialistico e a un trattamento farmacologico con amminoglicoside (amikacina) dosata per la funzione renale (FR) e carbapenemici, seguendo l'orientamento di un antibiogramma specifico. II paziente, negli ultimi mesi, inoltre, ha avuto una tendinite da chinolonico, antibiotico usato nei primi tre episodi infettiv .Il paziente ha mantenuto una virologia negativa per $\mathrm{CMV}$ e BK/JC durante tutti i ricoveri. L'ultimo episodio settico con presenza di Pseudomonas aeruginosa nelle urine (sensibile all'amikacina) si è arricchito con un'emocoltura positiva a Enterococcus faecium (sensibile al carbapenemico) con bi-terapia (amikacina+carbapenemico) protratta per 16 giorni con creatinina stabile e valutazione urologica per eventuale nefrectomia "selettiva". Alla consulenza urologica con la negativizzazione degli indici infiammatori (arrivati fino a 200 di PCR C-reattiva e $35.000 \mathrm{~GB}$ ), una creatinina arrivata a circa $2 \mathrm{mg} / \mathrm{dL}$ ma stabile e la scelta della nefrectomia del rene nativo poco selettiva hanno 
deposto per un atteggiamento attendista, una modifica della terapia IS con riduzione e, poi, arresto del MPA e un mantenimento in terapia con tacrolimus e corticosteroide. Attualmente, il paziente è apiretico, con FR stabile e tassi ematici di tacrolemia tra 6 e $8 \mathrm{mg} / \mathrm{dL}$.

Conclusioni: Le infezioni batteriche nel paziente trapiantato sono molto frequenti, soprattutto nel primo trimestre del post trapianto. Alcuni agenti batterici sono in grado di determinare quadri settici talmente gravi che possono determinare la necessità di utilizzare antibiotici anche ad alta nefrotossicità, pur di risolvere la sepsi. Una buona gestione di questi stessi farmaci a dosi corrette per FR e con una buona idratazione può, comunque, salvaguardare il paziente, la funzionalità renale e, nello specifico, il graft.

\section{DIALISI DOMICILIARE: PASSATO E FUTURO}

\section{5}

\section{DIALISI DOMICILIARE: PASSATO E FUTURO}

Mazzacani $P$.

Missionario volontario Fundacion Coen, Chinandega, Nicaragua

La dialisi domiciliare ha avuto un grande sviluppo in alcune delle grandi città italiane negli anni ' 80 , in risposta ai bisogni della popolazione e al contemporaneo limitato numero di servizi dialisi ospedalieri e sul territorio. L'aumento dei servizi dialisi e l'aumentare dell'età della popolazione dialitica hanno progressivamente lasciato posto a una maggiore "medicalizzazione" del trattamento, fino a una scomparsa della metodica, che solo in questi ultimi anni ha avuto una rinascita. Sarà presentata una panoramica degli obiettivi assistenziali, dei criteri dell’organizzazione dei servizi e della specificità del ruolo professionale rispetto al training e al monitoraggio del trattamento dialitico a domicilio negli anni ' 80 .

\section{2}

EMODIALISI DOMICILIARE: RIPRENDERE IN MANO LA PROPRIA VITA Miniscalco T.

S.C. Nefrologia e Dialisi, Ospedale S. G. Bosco, ASL TO2, Torino

Introduzione: In Piemonte, nei primi anni '70, il San G. Bosco mise a disposizione la possibilità di trattamento emodialitico a domicilio. A inizio anni '80, il centro contava circa 100 pazienti in trattamento. Successivamente, la crescente disponibilità di posti dialisi in nuovi centri, associata all'incremento dell'età media e a condizioni di rischio clinico nei pazienti all'ingresso, causò una progressiva contrazione della diffusione dell'emodialisi domiciliare. Incentivi Regionali ed evoluzione tecnologica dei monitor sembrano poter porre le basi per una ripresa.

Obiettivi: Dimostrare di mantenere i noti vantaggi dell'emodialisi riguardo alla buona qualità di vita, alla riabilitazione socio-lavorativa del paziente e alla riduzione dei costi diretti e indiretti del trattamento, ma permettendo anche una loro ottimizzazione.

Materiali e metodi: Revisione della casistica relativa a un pool di 15 P.A. in emodialisi domiciliare.

Discussione: L'emodialisi domiciliare sembra migliorare la qualità di vita dei pazienti e la disponibilità di tempo per il lavoro.

Risultati: L'E. D. sembra garantire buoni risultati di qualità di vita, di riabilitazione socio-lavorativa e di flessibilità degli schemi di trattamento.

\section{4}

EMODIALISI DOMICILIARE AL CAL: UN'ALTRA ALTERNATIVA DI VITA Paganini R. ${ }^{1}$, Aroldi R. ${ }^{1}$, Cosmai L. ${ }^{2}$

${ }^{1}$ Infermiere, U.O. Nefrologia e Dialisi Presidio Ospedaliero Oglio Po, A.S.S.T., Cremona; ${ }^{2}$ Nefrologo, U.O. Nefrologia e Dialisi Presidio Ospedaliero Oglio Po, A.S.S.T., Cremona

Introduzione: L'Emodialisi Extracorporea Domiciliare (HHD) è un'alternativa poco nota nel panorama dei trattamenti dialitici sostitutivi. Questo tipo di modalità, già utilizzato in altri Paesi e in molte Regioni italiane, può offrire significativi vantaggi in termini di outcome clinici, ma, soprattutto, di qualità di vita.
Metodologia: Nel nostro CAL, è iniziato un progetto di HDD attraverso il sistema NxStageSistemOne di Spindial. II progetto ha previsto inizialmente la presenza in sede di uno specialist per l'addestramento di un'infermiera, di un paziente e del suo care giver contemporaneamente. Dopo il primo paziente, il progetto è proseguito solo con il personale infermieristico e con il nefrologo referente sia per l'addestramento del secondo paziente che per il follow up. Risultati: Abbiamo ottenuto una migliore efficienza dialitica con riduzione della terapia domiciliare per entrambi i pazienti. Inoltre, la disponibilità infermieristica e medica 24 ore su 24 permette ai pazienti e ai care giver di avere un supporto tecnico assistenziale e medico continuo. II follow up con regolari visite e controlli mensili monitorizza l'andamento del trattamento e la prescrizione dialitica.

Conclusioni: Il progetto ha permesso ai pazienti di avere maggiore libertà di movimento nella vita quotidiana e una migliore compliance clinica. Ha permesso un riavvicinamento alla famiglia. Per noi infermieri è stata una crescita professionale e personale. La tecnologia ha fatto il resto.

044

\section{TELEMEDICINA: EMODIALISI DOMICILIARE}

Carbone $G$.

Unità Operativa di Nefrologia, Dialisi e Trapianto, Policlinico di Bari, Bari

Introduzione: Con la tele dialisi il trattamento emodialitico domiciliare è controllato attraverso una Control-Room, con la presenza di un infermiere e di un medico.

Tale sistema mette in relazione paziente e ospedale mediante un servizio di prevenzione, telemedicina e diagnosi collaborativa.

Metodologia: La digitalizzazione crea un dialogo tra dispositivi locali di controllo remoto e servizi informativi ospedalieri, usando apparecchi comuni, disponibili a tutti. SMART-HEALTH 2.0 PUGLIA.

Risultati: II risultato non è uno, ma sono davvero tanti i risultati di cui beneficiano diversi pazienti. Una medicina sempre più a contatto con il paziente, il quale si trova a essere protagonista del processo di cure e non solo utente passivo.

006

LA MORTE E IL MORIRE DEI PAZIENTI IN DIALISI: LA FASE DEL FOCUS GROUP DI UN' INDAGINE QUALITATIVA SULLA PERCEZIONE DEGLI INFERMIER

de Barbieri I. ${ }^{1}$, Bovo L. ${ }^{2}$, Beltrandi R. ${ }^{3}$, Burrai F. ${ }^{4}$

${ }^{1}$ Azienda Ospedaliera Universitaria di Padova, Padova; ${ }^{2}$ Libera professionista, Padova; ${ }^{3}$ Azienda Ospedaliera Universitaria di Bologna, Bologna; ${ }^{4}$ Governo clinico, Qualità, Formazione. Azienda Ospedaliera Universitaria di Bologna, Bologna

Introduzione: Negli ultimi dieci anni, il progresso medico e tecnologico ha portato a un miglioramento della dialisi e delle terapie di supporto sia in termini di sicurezza sia in termini di accessibilità. II risultato di questo perfezionamento è stato il prolungamento della vita di milioni di persone con patologie renali croniche (Cohen \& Germain, 2004). Quasi la metà di queste persone ha più di 65 anni e molte hanno sviluppato o sviluppano altre patologie come il diabete e malattie cardiovascolari e cerebrovascolari. Nonostante le terapie di supporto riescano, in molti casi, a garantire una buona qualità di vita, un numero crescente di decessi avviene per la decisione dei pazienti di sospendere queste terapie. Questa decisione emerge dal desiderio dei pazienti di porre fine alla continua e prolungata sofferenza e per la possibilità di morire dignitosamente e senza dolore (Cohen, Germain, Poppel, Woods, Pekow \& Kjellstrand, 2000). Attualmente, in Italia, esiste un solo centro che ha attivato un servizio di cure palliative specifiche per pazienti in insufficienza renale terminale. Questa ricerca ha, come obiettivo, quello di esplorare gli aspetti che riguardano la morte di pazienti in trattamento dialitico, come la decisione di sospendere il trattamento renale sostitutivo, la qualità della morte e il ruolo delle cure palliative per questa tipologia di pazienti, attraverso la percezione degli infermieri di dialisi.

Metodologia: La ricerca ha un approccio qualitativo. All'interno dell'Azienda Ospedaliero Universitaria di Bologna è stato organizzato un focus group, di durata non superiore alle 2 h, con 5 infermieri, che lavorano da almeno 5 anni in emodialisi e dialisi peritoneale, provenienti da differenti ospedali italiani. La partecipazione è stata volontaria, garantendo l'anonimato dei dati raccolti e della seduta. Ai partecipanti è stato chiesto di firmare il consen- 
so informato sulla partecipazione allo studio e l'informativa sulla privacy e di compilare una scheda anonima con dati anagrafici. II responsabile dello studio ha guidato il focus group attraverso un'intervista semi strutturata della quale i partecipanti non avevano precedente conoscenza. Un co-Autore dello studio ha osservato e annotato i particolari che emergevano durante l'incontro e le caratteristiche del gruppo. La seduta è stata registrata e i dati della seduta sono stati raccolti e conservati dal responsabile dello studio. Alla conclusione della ricerca, la registrazione verrà cancellata.

Conclusioni: In uno studio qualitativo sulla percezione che hanno gli infermieri sulla morte e sul morire dei pazienti in dialisi, la fase del focus group, la sua gestione e la sua conduzione sono fondamentali per una raccolta dati di alta qualità. I dati raccolti nel focus group verranno successivamente sistematizzati e analizzati in base a un framework specifico fenomenologico.

\section{0}

VALUTAZIONE DELL'EMPATIA NEGLI OPERATORI SANITARI DELLA U.O.C. DI NEFROLOGIA E DIALISI DELL'AREA VASTA 5 DI ASCOLI PICENO E SAN BENEDETTO DEL TRONTO

Narcisi D., Norcini S., Rossi L., Squarcia C., Tancredi A.

U.O.C. di Nefrologia e Dialisi, Area Vasta 5 Ascoli Piceno e San Benedetto del Tronto, Ascoli Piceno

Introduzione: L'abilità empatica è considerata un'attitudine fondamentale nella professione sanitaria. In particolare, le malattie croniche come l'uremia hanno un impatto profondo sulla vita delle persone, che devono riorganizzare radicalmente le proprie abitudini, le relazioni e l'attività lavorativa. Il personale sanitario che opera con i pazienti uremici ha il compito di accompagnare pazienti e familiari per molti anni, aiutandoli a mantenere sia un buono stato di salute sia la miglior qualità di vita possibile. È importante pertanto sviluppare nel tempo, oltre che capacità tecnico-scientifiche, anche capacità di relazione, come l'empatia. L'obiettivo di questo lavoro è di valutare la tendenza empatica negli operatori sanitari dell'U.O.C. di Nefrologia e Dialisi dell'Area Vasta 5.

Materiali e metodi: Nel nostro studio osservazionale, per la valutazione della tendenza empatica è stata somministrata la Balanced Emotional Empaty Scale (BEES) di A. Meharabian, nella versione validata in italiano, a un totale di 60 operatori di età compresa tra i 25 e i 65 anni. È stata valutata la differenza di empatia tra operatori di sesso maschile e femminile e tra fasce d'età (25-45 aa/46-65 aa). Sono stati, inoltre, valutati 5 facet ottenuti dalla somma di risposte fornite a gruppi di item, che ci hanno consentito di definire e di interpretare meglio il punteggio totale.

Risultati: II livello di tendenza empatica è risultato buono (la media della tendenza empatica del campione esaminato è di 29.02); gli operatori sanitari di sesso femminile hanno, tuttavia, ottenuto un punteggio di tendenza empatica superiore (Maschi 25.45 - Femmine 29.82). Si evidenzia, inoltre, come sia maggiore la tendenza empatica negli operatori, sia maschi che femmine, di fascia d'età superiore (M, F 25/45 = 23, M, F 46/65 = 37). I valori ottenuti sono sovrapponibili alle medie nazionali e internazionali, sia per il valore assoluto che per i valori di fasce di tendenza empatica.

Conclusioni: Sebbene lo studio osservazionale si sia svolto su un campione relativamente esiguo, ci ha permesso di valutare la nostra abilità empatica nella relazione professionale con il paziente uremico, consentendoci di individuare eventuali aree di carenza relazionale e quindi promuovere corsi di potenziamento, o aree di fatica da compassione, derivanti sia da personali caratteristiche e sensibilità, sia dall'eccessiva e continuativa esposizione emozionale compassionevole. Lo scopo ultimo sarà quello di organizzare, come prosecuzione, gruppi di discussione e interventi formativi tra operatori, con la guida di un esperto di dinamiche relazionali, con l'obiettivo di migliorare la gestione di situazioni relazionali complesse.

\section{2}

STRATEGIE ED EFFICACIA DELLA MEDICINA NARRATIVA NEI PAZIENT NEFROLOGICI: UNA REVISIONE DELLA LETTERATURA

Mettifogo M. ${ }^{1}$, Burrai $\mathrm{F}^{2}$, Zampieri C. ${ }^{1}$, Dorigo V. ${ }^{3}$

${ }^{1}$ Dipartimento di Nefrologia, Dialisi e Trapianto Ospedale S. Bortolo, Vicenza; ${ }^{2}$ Azienda Ospedaliero Universitaria di Bologna, Bologna; ${ }^{3}$ U.O.S Dialisi Ospedale di Dolo, Venezia

Introduzione: Si è diffuso da qualche decennio il concetto di Narrative Based Medicine (NBM), sorto dall'esigenza di avvicinare gli aspetti bio-medici della malattia e della cura a quelli psico-sociali, che fanno riferimento ai vissuti ad essi collegati.

Scopo: L'obiettivo è di individuare e sintetizzare le evidenze e i concetti della NBM in campo Nefrologico.

Metodi: È stata eseguita una revisione delle letteratura di studi consultando 6 banche dati.

Risultati: Sono stati inclusi 35 articoli, di cui nessuno è di tipo quantitativo.

Discussione: L'approccio narrativo applicato ai contesti operativi nefrologici cerca di far luce sui vissuti legati alla malattia renale, nei suoi diversi stadi, raccogliendo, attraverso interviste strutturate e semi strutturate, le storie di pazienti, familiari e sanitari. I topic principali sono: 1) "Liminalità", intesa come situazione di incertezza e passaggio, che caratterizza le esperienze di diagnosi incerta, di cura palliativa, di attesa e di sospensione della propria storia di vita; 2) "Indicibile", riferito a tutti quegli aspetti che non trovano spazio di espressione nella relazione di cura, come la convivenza con il rischio di morire ed esperienze difficili da verbalizzare, come tutto ciò che è legato al mistero e alla fede e tutto ciò che è inspiegabile. Nelle interviste narrative ai nefrologi e agli infermieri, emergono topic importanti, come una "coscienza turbata in situazioni eticamente difficili" e il ruolo di "protagonista indesiderato" derivato da situazioni problematiche di dialisi peritoneale e domiciliare.

Conclusioni: Da questa revisione è emersa l'importanza di esplorare gli aspetti inquietanti della vita delle persone affette da IRC, la cui conoscenza da parte de professionisti della salute può contribuire a migliorare qualità ed efficacia della relazione terapeutica e ad aiutare il paziente a non sentirsi isolato ma al centro del suo processo di cura. La Medicina Narrativa si rivolge anche ai sanitari, permettendo di indagare i loro vissuti, caratterizzati da un frequente contatto con situazioni di sofferenza e di disagio. La metodologia dell'approccio narrativo in ambito nefrologico potrebbe essere sostenuta dalla ricerca sulle capacità terapeutiche che la narrazione stessa può avere nella relazione di cura.

011

LA PERCEZIONE DEL MALESSERE E DEL DOLORE CORRELATI ALLA TIPOLOGIA DI ASSISTENZA RICEVUTA NEL PAZIENTE DIALIZZATO Neagu N., Rosetto Casel P., Talaia M.

Università degli Studi di Torino, Torino

Introduzione: L'obiettivo dello studio è la rilevazione della percezione de malessere e del dolore nei pazienti sottoposti a emodialisi e assistiti con modelli assistenziali diversi.

Metodologia: La ricerca è stata effettuata su due gruppi composti rispettivamente da 56 e 60 pazienti, quest'ultimo assistito tramite il modello primary nursing. Per raccogliere i dati, si è predisposto un questionario con domande inerenti la dialisi, le problematiche più frequenti ad essa correlate e quanto queste incidano sulla vita del paziente.

Risultati: I dati indicano importanti differenze nella percezione del dolore e de malessere fra i due gruppi. Nel gruppo di controllo risulta che il $43 \%$ dei pazienti prova più malessere rispetto agli anni passati mentre, nel gruppo primary nursing, tale percentuale è ridotta al $17 \%$. La percezione della salute è stata valutata con 7.1 su una scala da 1 a 10 (10 = stato di salute ottimo) nel gruppo primary nursing e con 5.5 nel gruppo di controllo (stessa scala). La soddisfazione dei pazienti rispetto alla presa in carico da parte degli infermieri e alla comunicazione con essi risulta di estrema importanza nella gestione del malessere e del dolore percepiti nei due gruppi con frequenza e intensità diverse.

Conclusioni: Il primary nursing permette una migliore presa in carico con maggiore soddisfazione per l'assistito e si riflette anche nella percezione della salute, del malessere e del dolore.

017

\section{UTILIZZO DELLA MUSICA IN EMODIALIS}

Micheluzzi V.

RSA Villa della Salute, Minerbio, Bologna

Gli infermieri sono i sanitari più vicini ai pazienti, perché interagiscono quotidianamente e maggiormente con essi e hanno, quindi, a che fare spesso con le loro preoccupazioni e con i bisogni di salute e di cura legati a diversi contesti, alcuni dei quali complessi come quello emodialitico. Molte persone, quando vengono sottoposte al trattamento emodialitico, hanno bisogno non solo di un approccio tecnologico ma anche olistico. Tra gli interventi olistici, la musica presenta numerose evidenze scientifiche sui suoi effetti sul benessere, sulla qualità della vita e sulla riduzione dello stress. La musicoterapia è 
un tipo di intervento non farmacologico, a basso costo per l'utente, personalizzabile, facilmente usufruibile e dotato di ripetibilità infinita grazie alla tecnologia attuale, facilmente maneggevole e utilizzabile e friendly per l'applicazione pratica. Attualmente, esistono, infatti, oltre che una serie di cuffie e auricolari diversi per ogni esigenza individuale, MP3 player, walkman, i-pod e presidi ultramoderni come i music-pillow, ovvero i cuscini musicali dotati di speaker integrati che possono connettersi direttamente alla fonte di ascolto utilizzata, che sia una radio, un $\mathrm{mp3}$ o la televisione. Gli infermieri, introducendo l'uso della musica in emodialisi, potrebbero ridurre lo stress, migliorare la qualità di vita e favorire il benessere dei pazienti.

\section{0}

\section{RIDUZIONE DELL'IMPATTO AMBIENTALE: GESTIONE} MULTIDISCIPLINARE DEI RIFIUTI SPECIALI IN UN CENTRO DIALISI Trombia L. ${ }^{1}$, Galli D. ${ }^{2}$, Moretti M. ${ }^{1}$, Pizzo A. ${ }^{1}$, Pelliccia F. ${ }^{3}$, Parisotto M.T. ${ }^{3}$ ${ }^{1}$ NephroCare Italia, Napoli, Italia; ${ }^{2}$ NephroCare Italia, Centro NephroCare Only Dialysis, Fiumicino, Roma, Italia; ${ }^{3}$ Fresenius Medical Care, NephroCare Coordination, Bad Homburg, Germania

Introduzione: Le Aziende che gestiscono i servizi di dialisi sono oggetto di analisi da parte delle autorità sanitarie e dei relativi investitori, considerando che i rifiuti rappresentano una parte significativa dei costi complessivi e rappresentano un elevato rischio ambientale. Nel 2014, è stato realizzato un progetto di miglioramento per la riduzione del carico ambientale dell'unità di dialisi, fissando un target interno di $1.0 \mathrm{~kg}$ a trattamento per i rifiuti contaminati dal sangue. Dopo un'attenta analisi di tutte le attività, è stato richiesto al personale di prestare particolare attenzione nell'utilizzo delle risorse impiegate e per ridurre l'impatto ambientale del centro di dialisi, per renderlo ecologicamente ed economicamente sostenibile.

Metodologia: È stata effettuata un'adeguata formazione al personale sanitario sulle procedure da rispettare nell'ambiente di lavoro, sulla corretta separazione dei rifiuti e sulla loro riduzione, seguita da un attento e continuo monitoraggio.

Risultati: Nel 2014, abbiamo raggiunto le seguenti riduzioni rispetto al 2013: Dicembre 2013: $1.20 \mathrm{~kg} /$ trattamento dei rifiuti contaminati da sangue; Giugno 2014: 1.12 kg/trattamento dei rifiuti contaminati da sangue; Novembre 2014: $0.98 \mathrm{~kg} /$ trattamento dei rifiuti contaminati da sangue.

Conclusioni: La formazione continua e la sensibilizzazione del personale infermieristico sono essenziali per migliorare il contenimento della produzione dei rifiuti contaminati da sangue. Inoltre, attraverso una valida pianificazione delle attività, una precisa attuazione delle procedure di gestione dei rifiuti e un monitoraggio continuo, abbiamo ottenuto una riduzione di $0.20 \mathrm{~kg}$ di rifiuti/trattamento, limitando gli sprechi in ogni fase del processo di dialisi e contenendo i costi.

\section{8}

\section{EMODIALISI QUOTIDIANA: STRATEGIE LOGISTICHE PER L'ABBATTIMENTO DEI COSTI}

Bartoccini J., Cannizzo V.

Ambulatorio di Emodialisi, Ospedale Santa Maria della Misericordia, Perugia

Introduzione: Molti studi sostengono che, aumentando il numero di dialisi a 5-6 volte a settimana, si migliorano la qualità di vita e la sopravvivenza rispetto alla dialisi trisettimanale.

L'accesso a queste modalità di dialisi è rimasto limitato per l'elevato costo dei trattamenti e dei trasporti.

Metodologia: Nello studio sono state eseguite delle simulazioni su 100 pazienti espletando 10 prove ciascuno. Questi sono stati, quindi, riuniti in gruppi. tenendo conto della capacità dei mezzi di trasporto. Sono state eseguite simulazioni logistiche in un gruppo di 10 pazienti del nostro centro dialisi.

Risultati: Dimostrano un risparmio di corse del $60 \%$ e di chilometri percorsi del $30 \%$, evidenziando come la variabile trasporti, se ben gestita, possa ridurre i costi della dialisi.

Conclusioni: L'obiettivo dello studio era la comparazione di km percorsi e numero corse rispetto ai costi, per valutare quale metodo sia più opportuno nelle varie realtà. Dalle simulazioni effettuate si è visto che un'accorta gestione della spesa legata al trasporto dei pazienti abbatte i costi dei trasporti.
031

INNOVAZIONI TECNOLOGICHE IN UN CENTRO DI NEFROLOGIA E DIALISI: RUOLO DEL PERSONALE SANITARIO INFERMIERISTICO E MEDICO

Liani V. ${ }^{1}$, Melchiorre A. ${ }^{1}$, Trabassi E. ${ }^{1}$, Cacciagrano M.R. ${ }^{2}$

${ }^{1}$ Centro Nefrologia e Dialisi, P.O. "San Massimo", Penne, Pescara; ${ }^{2}$ Ufficio Infermieristico, P.O. "San Massimo”, Penne, Pescara

Introduzione: La crescente diffusione di Diabete Mellito tipo 2 (T2DM) rappresenta un grave problema di salute pubblica e si associa a molteplici complicanze invalidanti tra cui patologie ischemiche agli arti inferiori, nonostante il controllo accurato del glucosio. Il decorso dei pazienti con T2DM in emodialisi è ancora peggiore. Malati con T2DM sottoposti ad amputazione maggiore mostrano complicanze severe e richiedono maggiore assistenza; il decorso post-operatorio è più difficile e la prognosi è caratterizzata da ridotta sopravvivenza e da una qualità della vita compromessa.

Recenti ricerche hanno evidenziato, in malati con T2DM e ulcere difficili, che l'esposizione a campi elettrostatici o a campi elettromagnetici pulsati induce un'accelerazione della guarigione delle ferite agli arti inferiori associata a una riduzione sensibile del dolore e a una conseguente maggiore autonomia e a un generale migliorato benessere. All'effetto non è estraneo il contributo di una migliorata performance dell'endotelio. I microcircolo garantisce un apporto non solo quantitativo di nutrienti ma anche qualitativo.

Nel quotidiano, l'assenza di dolore condiziona positivamente l'autonomia individuale. II T2DM è una patologia multiorgano ed è gravato da limitazioni progressivamente ingravescenti che riducono il grado di autonomia. Appare evidente che, a oggi, la prevenzione, la profilassi e le procedure finalizzate al ripristino della funzionalità clinica hanno migliorato la qualità di vita ma non la prognosi individuale. Durante il ciclo PESF, oltre alla riduzione del dolore percepito, sono stati segnalati aumento del metabolismo basale, riduzione del peso corporeo e, a livello del microcircolo, maggiore dinamismo del microcircolo stesso o "vasomotion".

Metodologia: Ritenendo che alcuni dei fenomeni segnalati siano attribuibili, almeno in parte, a un'aumentata performance del microcircolo, abbiamo cercato di cogliere, in un gruppo di 30 individui con T2DM trattati con PESF, eventuali variazioni della qualità di vita percepita.

Come suggerito da Gordon, oltre alle limitazioni obiettive, il benessere percepito dipende da una vasta gamma di condizioni, tra cui la percezione e la gestione della propria salute, le libertà alimentari in rapporto a modelli $\mathrm{d}$ nutrizione, il grado di attività fisica, il grado di cognizione e percezione, il ritmo e la qualità del sonno-veglia e le relazioni sociali, che sono indubbiamente compromesse quando sussistono limitazioni.

A tale scopo, abbiamo adottato, attenendoci ai modelli suggeriti da "Gordon", apposite schede per il monitoraggio di variazioni dello stato di benessere e per cogliere la volontà a proseguire le cure nell'immediato o a riprenderle nel futuro.

La struttura dei modelli funzionali di Gordon ha un carattere olistico e si applica alla persona, in considerazione del fatto che le entità distinte vanno ricollegate all'unicità e alla complessità della persona stessa.

I metodi che abbiamo utilizzato per la raccolta dati sono stati: 1) l'osservazione, 2) il colloquio-intervista, 3) l'esame fisico e 4) la visione della documentazione clinica.

Risultati: Abbiamo notato una riduzione della pressione arteriosa sistolica e diastolica e, in alcuni, la necessità di adeguare la terapia ipotensiva. Le condizioni trofiche della pelle sono migliorate (in particolare le dimensioni delle ferite) e il colorito delle mucose è divenuto più roseo. Non abbiamo notato differenze sulla caduta dei capelli. La temperatura corporea non è variata. II peso corporeo è risultato ridotto. La volontà di svolgere le comuni attività della vita quotidiana (domestica e tempo libero) è migliorata. Le ore di veglia e di riposo e la percezione della qualità del proprio sonno sono migliorate.

Conclusioni: I dati raccolti, riportati su schede appositamente predisposte, oltre alla misurazione dei risultati prefissati, offrono delle variazioni minime ma, ricollegate tutte nell'unicità e nella complessità della persona stessa, giustificano ampiamente il miglioramento dello stato di benessere avvertito e riferito dai pazienti. È evidente che la riduzione del peso corporeo (non ottenuta con i precedenti tentativi) e della pressione arteriosa e, soprattutto, la significativa riduzione del dolore percepito, oltre all'aumento dell'attività fisica libera dal dolore, facilitano le relazioni e aumentano il benessere soggettivo. 


\section{LA TECNICA MENTE CORPO DELLA GUIDED IMAGERY}

\section{8}

L'ASSISTENZA OLISTICA IN NEFROLOGIA: INNOVAZIONE E FUTURO Burrai F. ${ }^{1}$, Benfenati $E^{2}{ }^{2}$, Ferretti S. $^{2}$

${ }^{1}$ Governo Clinico Qualità Formazione. Azienda Ospedaliero Universitaria di Bologna, Bologna; ${ }^{2}$ Studentessa $3^{\circ}$ Anno Infermieristica, Università di Bologna, Sede formativa AUSL di Bologna-BO2, Bologna

L'assistenza quotidiana si basa fondamentalmente sul modello riduzionistico-meccanicistico, con una base epistemologica di tipo cartesianonewtoniano. Questo modello frammenta l'individuo, identifica il paziente con la malattia e suddivide l'essere umano in parti anatomiche che, se non funzionanti, necessitano di essere "aggiustate", senza nessun legame con una componente mentale, la quale non è considerata come influente sugli esiti dell'intervento. Dunque, l'assistenza standard delega completamente solo all'applicazione di una tecnologia il potere di migliorare le condizioni di un paziente, introducendo una pratica meramente tecnico-esecutiva, codificata, ripetitiva e impersonale e, per il personale, nel tempo non gratificante, demotivante e stancante. Ma l'essere umano non è una macchina. Secondo il modello olistico, la persona non può essere ridotta semplicemente alle sue componenti organiche, ma, in base alla teoria dei sistemi, ogni essere vivente è un insieme che ha un significato diverso e maggiore rispetto alla semplice somma delle sue parti, e questa differenza è prodotta dalle relazioni tra le parti biologiche, psicologiche, spirituali connesse ai contesti sociali e fisico ambientali. Tale network comunica istantaneamente attraverso una comunicazione circolare e con feedback continui. Queste componenti influenzano ogni esito di qualsiasi intervento assistenziale. Da considerare, inoltre, che sempre più pazienti presentano una migliore preparazione culturale e non accettano di essere considerati semplicemente in maniera spersonalizzata, assistiti (to cure) in un ottica prevalentemente biologica. L'assistenza olistica ritiene fondamentale la considerazione della componente esistenziale della persona (to care), dei suoi valori, delle sue opinioni e delle sue credenze e delle sue risorse, trasformando il quotidiano routinario in un quotidiano sempre nuovo. Gli interventi olistici sono di carattere non farmacologico e si basano sulla connessione psico-neuro-endocrino-immunologica ed energetica. Gli interventi olistici applicabili e che presentano evidenze sono la guided imagery, il therapeutic touch, le tecniche di rilassamento con la respirazione, l'utilizzo della musica e del suono, l'arte e la comunicazione ipnotica. L'assistenza moderna in nefrologia dovrebbe introdurre progressivamente infermieri con competenze olistiche, per migliorare la qualità di vita e i diversi outcome fisiologici, psicologici e spirituali dei pazienti che assistono, in sinergia e complementarietà con gli interventi farmacologici e tecnologici. Per raggiungere tale obiettivo, è necessario programmare un percorso sistematico nazionale di formazione olistica, sia a livello di Master universitari sia a livello ECM, per creare in pochi anni un'assistenza nefrologica moderna e capace di rispondere in maniera personalizzata ai bisogni dei pazienti. 